\title{
Statistical study of foreshock cavitons
}

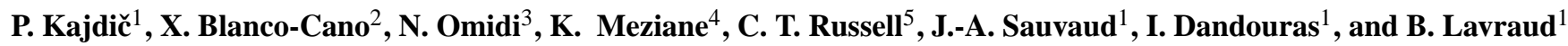 \\ ${ }^{1}$ Institut de Recherche en Astrophysique et Planétologie, University of Toulouse, UMR5277, CNRS, Toulouse, France \\ ${ }^{2}$ Instituto de Geofísica, Universidad Nacional Autónoma de México, Ciudad Universitaria, México D. F., México \\ ${ }^{3}$ Solana Scientific Inc., Solana Beach, CA, USA \\ ${ }^{4}$ Physics Department, University of New Brunswick, Fredericton, Canada \\ ${ }^{5}$ Institute of Geophysics and Planetary Physics, University of California, Los Angeles, CA, USA
}

Correspondence to: P. Kajdič (kajdic@gmail.com)

Received: 29 April 2013 - Revised: 30 August 2013 - Accepted: 8 November 2013 - Published: 6 December 2013

\begin{abstract}
In this work we perform a statistical analysis of 92 foreshock cavitons observed with the Cluster spacecraft 1 during the period 2001-2006. We analyze time intervals during which the spacecraft was located in the Earth's foreshock with durations longer than $10 \mathrm{~min}$. Together these amount to $\sim 50$ days. The cavitons are transient structures in the Earth's foreshock. Their main signatures in the data include simultaneous depletions of the magnetic field intensity and plasma density, which are surrounded by a rim of enhanced values of these two quantities. Cavitons form due to nonlinear interaction of transverse and compressive ultra-low frequency (ULF) waves and are therefore always surrounded by intense compressive ULF fluctuations. They are carried by the solar wind towards the bow shock. This work represents the first systematic study of a large sample of foreshock cavitons. We find that cavitons appear for a wide range of solar wind and interplanetary magnetic field conditions and are therefore a common feature upstream of Earth's quasiparallel bow shock with an average occurrence rate of $\sim 2$ events per day. We also discuss their observational properties in the context of other known upstream phenomena and show that the cavitons are a distinct structure in the foreshock.
\end{abstract}

Keywords. Magnetospheric physics (solar windmagnetosphere interactions) - Space plasma physics (shock waves; wave-particle interactions)

\section{Introduction}

The solar wind (SW) is a magnetized plasma that flows away from the Sun at supersonic speeds. On its way through the solar system this plasma encounters obstacles such as planets and planetary magnetospheres. When the solar wind hits a magnetosphere, it is slowed down, heated and deflected to flow around it. The deceleration and the heating occur mainly at the planetary bow shocks. The Earth's bow shock is a high Mach number shock with typical magnetosonic Mach number $\left(M_{\mathrm{ms}}\right) \sim 8$. It is also a collisionless shock, meaning that a free mean path for ion binary collisions $(\sim 1$ AU at heliospheric distance of $1 \mathrm{AU}$ ) is much larger than the size of the shock and its transition region. Due to its high Mach number, the Earth's bow shock is typically supercritical. This means that a large part of the solar wind's kinetic energy is dissipated by energizing and reflecting a small portion of its particles back upstream (e.g., Treuman, 2009).

The phenomena that exist upstream of the Earth's bow shock depend on the angle between the local-shock normal and the upstream interplanetary magnetic field (IMF): $\theta_{B n}$. The shock is labeled quasi-parallel or quasi-perpendicular, depending on whether the $\theta_{B n}$ is smaller or larger than $45^{\circ}$. The region upstream of the quasi-parallel bow shock is populated by hot ion populations and ultra-low frequency (ULF) magnetic field fluctuations with periods of $\sim 30$ s (Hoppe and Russell, 1981; Greenstadt et al., 1995). The ULF waves can appear as sinusoidal, transverse waves propagating almost parallel with respect to the upstream IMF, or they can be compressive, obliquely propagating fluctuations. The region upstream of the Earth's bow shock that is magnetically 
connected to it, is called the Earth's foreshock (Le and Russell, 1992a, b; Eastwood et al., 2005; Greenstadt et al., 1995).

In addition to ions and waves, there are also transient phenomena that populate the Earth's foreshock. Phenomena such as hot flow anomalies (HFAs) (Thomsen et al., 1986; Schwartz et al., 1995; Lucek et al., 2004; Zhang et al., 2010), density holes (Parks et al., 2006, 2008; Wilber et al., 2008) and foreshock cavities (Sibeck et al., 2001, 2002, 2008; Billingham et al., 2008; Schwartz et al., 2006) have been studied extensively in the past. Here we study another transient phenomenon, foreshock cavitons. These structures have first been described by Lin (2003), Lin and Wang (2005) and Omidi (2007) based on their hybrid simulation results. Lin (2003) and Lin and Wang (2005) referred to the cavitons as diamagnetic cavities, while Omidi (2007) still called them foreshock cavities. The name, cavitons, was used in later papers (e.g., Blanco-Cano et al., 2009, 2011) in which hybrid simulation results are compared to Cluster observations and in Kajdič et al. (2011) which is the first multispacecraft study of foreshock cavitons. Examples of simulated foreshock cavitons can be seen in Fig. 1. Panel a shows density normalized to the solar wind value from a hybrid simulation. Cavitons are seen as white regions, indicating low density values. Panels $\mathrm{b}$ and $\mathrm{c}$ show the magnetic field strength and plasma density as functions of time as seen by a virtual spacecraft located in point " $\mathrm{X}$ " on panel a. At the time of a caviton the values of $B$ and $n$ are strongly diminished.

Past numerical simulations predicted $\boldsymbol{B}$ field magnitude and density drops of $\sim 50 \%$ inside the cavitons, with respect to the ambient values. These drops would be larger for cavitons closer to the bow shock. A more moderate drop of $\sim 10 \%$ was predicted for solar wind bulk velocity. Simulated cavitons were also found to be surrounded by a rim of enhanced $B$ and $n$ values. The proposed formation mechanism for foreshock cavitons includes the nonlinear interaction of compressive, obliquely propagating and transverse, parallelpropagating ULF waves (Omidi, 2007).

Early hybrid simulations were performed for parallel IMF geometries and it was Blanco-Cano et al. (2011) who showed that foreshock cavitons could also be observed for non-radial IMF configurations. Lin (2003) and Lin and Wang (2005) also predicted that cavitons would eventually evolve into structures elongated along the $\boldsymbol{B}$ field lines and that their pressure pulses may perturb the magnetopause.

The observations in general agreed with the numerical predictions. Blanco-Cano et al. (2011) and Kajdič et al. (2011) reported $B$ and $n$ depressions between $\sim 40 \%$ and $\sim 50 \%$ inside the cavitons. Kajdič et al. (2011) showed that cavitons propagate sunward in the plasma frame of reference, but are carried antisunward by the SW. It was also shown by these authors that foreshock cavitons are always surrounded by compressive ULF fluctuations, which is consistent with their proposed formation mechanism.

Based on recent observations and hybrid simulations, it is believed that once foreshock cavitons reach and collide
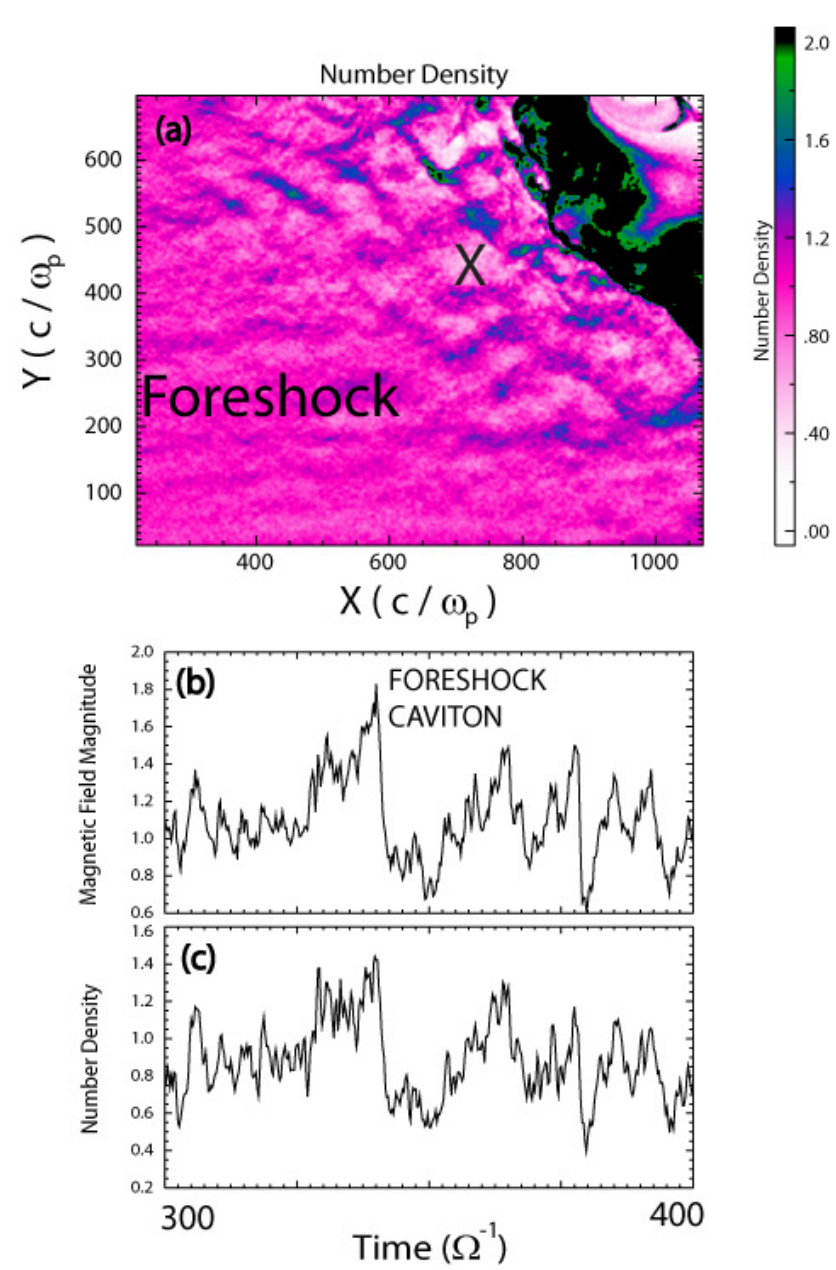

Fig. 1. (a) shows the density normalized to the solar wind value from a hybrid simulation run with Alfvénic Mach number of 11. The panel is zoomed around the quasi-parallel shock and ion foreshock which shows a number of cavitons identified as white colored regions. Note the color table is set to maximum of 2 in order to make the foreshock cavitons visible. The magnetosheath corresponds to the black region in the figure. (b) and (c) show the magnetic field strength and number density as a function of time measured by a simulated spacecraft located at point " $X$ " in (a) and illustrate the signatures of a foreshock caviton in the time series data.

with the bow shock, they produce another phenomenon, the so called spontaneous hot flow anomalies (SHFA, see Omidi et al., 2013a; Zhang et al., 2013). SHFAs seem to be an important part of shock dissipation processes and in turn impact the magnetosheath.

Few observations have been reported until now, and no systematic study of a larger sample of foreshock cavitons has been performed yet. In this paper, we study a sample of 92 foreshock cavitons that were found in the Cluster 1 data during the period 2001-2006. The full list of events is provided in Table 1. From our sample of events, we calculate the average values of their sizes, the magnitude of magnetic field 
and plasma-density depletions inside the cavitons and their durations in the data. We show that $B$ and $n$ inside the cavitons are much more correlated than in case of surrounding ULF fluctuations. We also show that cavitons are surrounded by compressive ULF fluctuations and are associated with diffuse ion populations. Finally, we estimate the occurrence rate of observable foreshock cavitons to be $\sim 2$ events per day.

Cavitons exist in regions also populated by intense ULF waves. In the data, these fluctuations appear immediately before and after the cavitons and persist for several minutes or even hours. All this makes the cavitons difficult to identify. One needs to be cautious in order to really distinguish foreshock cavitons from other phenomena in their surroundings. Many caviton candidates have been discarded during the selection process.

The phenomenon that observationally most resemble the foreshock cavitons are the foreshock cavities (Sibeck et al., 2001, 2002, 2008; Billingham et al., 2008, 2011). Due to apparent similarities there has been some doubt in the past whether the two phenomena are really different structures. However there are some important observational differences that enable us to distinguish between them. Cavitons will always be found in regions populated by compressive ULF waves. The suprathermal ion populations and the total (plasma + magnetic field) pressure inside them will be the same as in their immediate surroundings. Cavities, on the other hand, exhibit hot ion populations in their interiors, while in regions that surround them the distributions correspond either to pristine SW population or to field aligned ion beams. The total pressure inside the cavities exceeds the one in their surroundings.

We show here that foreshock cavitons are a common feature in the Earth's foreshock different from other foreshock phenomena. They appear for a large range of SW and IMF conditions. We demonstrate that $\boldsymbol{B}$ field magnitude and SW density inside the cavitons are highly correlated and we design a new criteria which enables us to distinguish the cavitons from the surrounding ULF fluctuations.

\section{Observations}

Cluster is a four-spacecraft mission in orbit around the Earth that provides magnetic field and plasma measurements in the near-Earth environment. The Cluster satellites carry several instruments onboard. Here we use the magnetic field data provided by the Fluxgate Magnetometer (FGM, Balogh et al., 2001) and the Cluster Ion Spectrometer (CIS, Rème et al., 2001). The FGM data are available in three time resolutions: $22 \mathrm{~s}^{-1}, 5 \mathrm{~s}^{-1}$ and 1 vector per spin ( $\left.4 \mathrm{~s}\right)$. The CIS-HIA instrument provides full, 3-D ion distributions and moments in the energy range between $5 \mathrm{eV}$ and $32 \mathrm{keV}$ with 1 spin time resolution. The HIA is composed of the "high-G" or "highsensitivity" (HS) and "low-g" or "low-sensitivity" (LS) sections. In the magnetospheric operational modes (MS), the
HIA performs a full energy sweep 32 times per spin, thereby accounting for the angular resolution of $11.25^{\circ}$. In the solar wind modes, the sweep is truncated above the energy of alpha particles whenever the "high-G" section faces the Sun. When the field of view of the "low-g" section is within $45^{\circ}$ centered on the solar wind direction, this section performs eight sweeps with $5.625^{\circ}$ angular resolution. Hence, the SW is detected only by the "low-g" side and only this data is used for the calculation of the SW moments.

Foreshock cavitons are identified as simultaneous depressions of $\boldsymbol{B}$ field magnitude and plasma density surrounded by a rim of enhanced values of these two quantities. We set a threshold of the minimum depressions of $B$ and $n$ to be $20 \%$ in order for the event to be taken into account. Also, these depressions have to be lower than the minimum level of surrounding ULF fluctuations. The foreshock cavitons in our sample also tend to be wider (i.e., last longer in time series) than the wavelengths of the surrounding ULF waves and are easily recognizable by eye as distinct features.

In order not to confuse cavitons with other foreshock phenomena, we require that (1) the plasma temperature inside the cavitons remains the same as in their surroundings, (2) there is no flow deviation inside the cavitons, and (3) there are no IMF discontinuities associated with the events.

It should be mentioned that hybrid simulations by Omidi et al. (2013a) have shown that close to the bow shock, the plasma temperature and velocity changes may be associated with the cavitons. Since these variations are not inherent to the cavitons and because they are also observed in association with other foreshock phenomena, such as HFAs and foreshock cavities, we discard caviton candidates that exhibited them. The sample is a consequence of our stringent selection criteria.

Figure 2 shows an example of a foreshock caviton that was observed on 26 April 2006. During this time, the Cluster spacecraft 1 was operating in a solar wind mode. The panels show (from top to bottom): magnetic field magnitude with $4 \mathrm{~s}$ time resolution in nanoteslas (nT), magnetic field components in geocentric solar ecliptic (GSE) coordinate system (nT), solar wind density $\left(\mathrm{cm}^{-3}\right)$, thermal pressure $(\mathrm{nPa})$, total solar wind velocity $\left(\mathrm{km} \mathrm{s}^{-1}\right)$, solar wind velocity components $\left(\mathrm{km} \mathrm{s}^{-1}\right.$ ), and CIS-HIA spectrogram (HS) for suprathermal ions and CIS-HIA spectrogram (LS) for solar wind ions. The two vertical red lines delimit the time interval during which the caviton was observed (from 09:15:56 UT to $09: 17: 12 \mathrm{UT}$ ). The duration of the caviton was $76 \mathrm{~s}$. The two small blue vertical lines mark the times of ion distributions shown in Fig. 3. We can see that the event is surrounded by a region populated with compressive ULF fluctuations of $\boldsymbol{B}$ field and plasma density. The average values of $B$ and $n$ in the surrounding medium during the presented time interval are $3.8 \mathrm{nT}$ and $5.1 \mathrm{~cm}^{-3}$, respectively. During the event, these two variables reach minimum values of $1.6 \mathrm{nT}$ and $1.8 \mathrm{~cm}^{-3}$. This represents a $58 \%$ drop in $\boldsymbol{B}$ field and $65 \%$ drop in $n$. The interior of the caviton is surrounded 
Table 1. List of foreshock cavitons in the sample. The columns contain the following information (from left to right): date and time of cavitons, their durations, their coordinates in the GSE coordinate system, operational mode of the spacecraft at time of observation of cavitons, magnitudes of depletions of $B$ and $n$ inside the events, SW Alfén number and SW alfvénic Mach number.

\begin{tabular}{|c|c|c|c|c|c|c|c|c|c|c|}
\hline $\begin{array}{l}\text { Date } \\
\text { DD/MM/YYYY }\end{array}$ & $\begin{array}{l}\text { Time [UT] } \\
\text { HH:MM:SS }\end{array}$ & $\begin{array}{l}\text { Duration [s] } \\
\text { MM:SS }\end{array}$ & $\begin{array}{r}x_{\mathrm{GSE}} \\
R_{\mathrm{E}}\end{array}$ & $\begin{array}{r}y_{\mathrm{GSE}} \\
R_{\mathrm{E}}\end{array}$ & $\begin{array}{r}z_{\mathrm{GSE}} \\
R_{\mathrm{E}}\end{array}$ & $\begin{array}{l}\text { Operational } \\
\text { mode }\end{array}$ & $\Delta B / B$ & $\Delta n / n$ & $\begin{array}{r}V_{\mathrm{A}} \\
\mathrm{km} \mathrm{s}^{-1}\end{array}$ & $M_{\mathrm{A}}$ \\
\hline $15 / 02 / 2001$ & 08:09:03 & 01:01 & 18.86 & 5.21 & 1.7 & SW & 0.55 & 0.70 & 87 & 5.9 \\
\hline $15 / 02 / 2001$ & $08: 41: 56$ & $02: 25$ & 18.87 & 5.08 & 1.31 & SW & 0.67 & 0.70 & 85 & 6.2 \\
\hline $15 / 02 / 2001$ & 08:50:08 & $02: 28$ & 18.87 & 4.95 & 1.18 & SW & 0.37 & 0.46 & 90 & 5.9 \\
\hline $15 / 02 / 2001$ & $09: 24: 22$ & $00: 44$ & 18.87 & 4.82 & 0.85 & SW & 0.53 & 0.50 & 87 & 6.0 \\
\hline $15 / 02 / 2001$ & 09:30:40 & $00: 42$ & 18.87 & 4.75 & 0.72 & SW & 0.47 & 0.50 & 98 & 5.3 \\
\hline $15 / 02 / 2001$ & $11: 10: 46$ & $00: 38$ & 18.77 & 4.36 & -0.12 & SW & 0.47 & 0.54 & 90 & 5.8 \\
\hline $21 / 02 / 2001$ & 21:49:20 & 01:19 & 14.27 & 7.93 & 4.94 & SW & 0.59 & 0.63 & 53 & 6.4 \\
\hline 08/04/2001 & 03:18:52 & $01: 36$ & 13.64 & 7.44 & -6.81 & SW & 0.51 & 0.60 & 90 & 5.0 \\
\hline 08/04/2001 & $03: 38: 27$ & $00: 54$ & 13.8 & 7.44 & -6.96 & SW & 0.29 & 0.38 & 91 & 5.1 \\
\hline $12 / 02 / 2002$ & 11:59:41 & 00:52 & 14.67 & 1.87 & -6.8 & SW & 0.49 & 0.50 & 88 & 5.1 \\
\hline $12 / 02 / 2002$ & 12:09:30 & 01:00 & 14.57 & 1.78 & -6.98 & SW & 0.61 & 0.60 & 88 & 5.1 \\
\hline $16 / 02 / 2002$ & $08: 33: 37$ & $00: 54$ & 16.25 & 5.91 & 4.68 & SW & 0.26 & 0.31 & 81 & 4.0 \\
\hline $16 / 02 / 2002$ & 09:33:28 & 01:04 & 16.73 & 5.8 & 4.15 & SW & 0.30 & 0.46 & 72 & 4.4 \\
\hline $21 / 02 / 2002$ & $06: 47: 31$ & 01:04 & 18.3 & 3.83 & 2.44 & SW & 0.44 & 0.33 & 97 & 4.7 \\
\hline 21/02/2002 & $07: 16: 27$ & $00: 57$ & 18.44 & 3.76 & 2.17 & SW & 0.35 & 0.48 & 94 & 4.7 \\
\hline 21/02/2002 & 07:58:11 & $00: 37$ & 18.6 & 3.61 & 1.82 & SW & 0.35 & 0.38 & 99 & 4.5 \\
\hline $21 / 02 / 2002$ & 08:01:43 & 00:38 & 18.6 & 3.61 & 1.82 & SW & 0.27 & 0.35 & 99 & 4.5 \\
\hline 21/02/2002 & $17: 30: 12$ & $00: 27$ & 18.29 & 1.3 & -3.69 & SW & 0.37 & 0.29 & 81 & 5.0 \\
\hline 09/03/2002 & $13: 03: 30$ & $00: 58$ & 14.1 & 7 & 0.94 & SW & 0.51 & 0.63 & 57 & 6.7 \\
\hline 09/03/2002 & $13: 25: 00$ & $01: 13$ & 14.4 & 6.8 & 0.88 & SW & 0.68 & 0.71 & 59 & 6.8 \\
\hline $16 / 03 / 2002$ & $13: 03: 15$ & $01: 12$ & 11.21 & 7.91 & 0.34 & MS & 0.62 & 0.38 & 56 & 5.4 \\
\hline $16 / 03 / 2002$ & $13: 38: 08$ & $01: 16$ & 11.78 & 7.75 & 0.18 & MS & 0.57 & 0.59 & 57 & 5.3 \\
\hline $22 / 05 / 2002$ & $11: 27: 20$ & 00:41 & 4.7 & 4.13 & -16.45 & MS & 0.41 & 0.47 & 45 & 9.0 \\
\hline 03/02/2003 & 10:58:04 & 01:00 & 14.23 & 9.4 & 3.19 & SW & 0.34 & 0.41 & 69 & 6.9 \\
\hline $04 / 02 / 2003$ & 09:02:24 & 01:40 & 13.32 & 3.01 & -8.58 & SW & 0.52 & 0.49 & 100 & 5.5 \\
\hline $04 / 02 / 2003$ & 09:06:48 & 01:08 & 13.22 & 3.01 & -8.71 & SW & 0.36 & 0.48 & 99 & 5.5 \\
\hline 04/02/2003 & $10: 30: 54$ & $00: 38$ & 11.88 & 2.26 & -9.09 & SW & 0.23 & 0.24 & 124 & 4.4 \\
\hline 04/02/2003 & $10: 34: 48$ & 00:28 & 12.23 & 2.13 & -9.09 & SW & 0.26 & 0.34 & 119 & 4.6 \\
\hline 05/02/2003 & $22: 35: 20$ & $02: 31$ & 15.75 & 8.67 & 1.71 & SW & 0.48 & 0.43 & 105 & 5.0 \\
\hline 08/02/2003 & $12: 49: 16$ & $00: 55$ & 17.41 & 7.06 & -1.41 & SW & 0.35 & 0.39 & 82 & 5.5 \\
\hline 09/02/2003 & $02: 35: 41$ & 01:06 & 13.92 & 2.11 & -8.5 & SW & 0.73 & 0.75 & 78 & 5.6 \\
\hline $15 / 02 / 2003$ & $14: 25: 20$ & $00: 55$ & 17.93 & 5.22 & -0.44 & SW & 0.28 & 0.30 & 82 & 7.1 \\
\hline $16 / 02 / 2003$ & $01: 29: 46$ & 00:50 & 16.54 & 1.9 & -6.68 & SW & 0.40 & 0.50 & 97 & 5.7 \\
\hline $16 / 02 / 2003$ & 04:11:03 & 00:44 & 15.15 & 0.94 & -7.88 & SW & 0.36 & 0.50 & 86 & 6.4 \\
\hline $25 / 02 / 2003$ & $07: 03: 27$ & $01: 44$ & 18.63 & 1.03 & -3 & SW & 0.39 & 0.52 & 41 & 10.0 \\
\hline $27 / 02 / 2003$ & $21: 49: 27$ & 01:01 & 17.17 & -1.29 & -6.3 & SW & 0.33 & 0.32 & 82 & 5.9 \\
\hline 06/03/2003 & $23: 15: 32$ & 00:58 & 17.58 & -3.03 & -5.26 & SW & 0.39 & 0.42 & 63 & 7.4 \\
\hline 08/03/2003 & $14: 02: 02$ & 01:19 & 14.48 & 1.19 & 5.34 & SW & 0.71 & 0.64 & 52 & 7.7 \\
\hline $15 / 03 / 2003$ & $16: 21: 57$ & $00: 57$ & 13.81 & -0.25 & 5.77 & MS & 0.74 & 0.68 & 100 & 6.1 \\
\hline $22 / 03 / 2003$ & $18: 04: 25$ & $00: 52$ & 12.38 & -1.25 & 6.43 & MS & 0.84 & 0.85 & 91 & 6.0 \\
\hline 23/03/2003 & $01: 22: 43$ & $00: 45$ & 16.66 & -4.44 & 2.64 & SW & 0.36 & 0.52 & 62 & 8.9 \\
\hline 23/03/2003 & $03: 17: 54$ & 01:02 & 17.18 & -5.14 & 1.49 & SW & 0.64 & 0.68 & 79 & 7.2 \\
\hline 03/04/2003 & $17: 23: 11$ & $00: 47$ & 13.09 & -4.9 & 5.55 & MS & 0.39 & 0.46 & 89 & 5.0 \\
\hline 03/04/2003 & $23: 34: 10$ & $00: 29$ & 15.55 & -8.07 & 2.14 & SW & 0.29 & 0.32 & 59 & 7.3 \\
\hline 08/04/2003 & 15:07:58 & 00:56 & 14.22 & -8.06 & 3.65 & SW & 0.24 & 0.28 & 63 & 6.6 \\
\hline 08/04/2003 & $15: 13: 07$ & $00: 35$ & 14.24 & -8.11 & 3.6 & SW & 0.20 & 0.27 & 62 & 6.7 \\
\hline $22 / 04 / 2003$ & $21: 35: 56$ & $00: 55$ & 11.83 & -11.22 & 3.66 & SW & 0.49 & 0.67 & 91 & 5.5 \\
\hline $22 / 04 / 2003$ & $22: 41: 22$ & 01:30 & 12 & -11.84 & 3.04 & SW & 0.50 & 0.57 & 79 & 6.4 \\
\hline 27/04/2003 & $16: 37: 02$ & 00:52 & 10.97 & -12.61 & 3.15 & SW & 0.79 & 0.80 & 74 & 6.3 \\
\hline 07/05/2003 & $05: 53$ & $00: 31$ & 8.25 & -10.75 & 5.72 & MS & 0.54 & 0.61 & 120 & 5.6 \\
\hline $11 / 05 / 2003$ & $20: 51: 54$ & $00: 48$ & 7.62 & -13.42 & 4.4 & MS & 0.73 & 0.74 & 119 & 5.4 \\
\hline $11 / 05 / 2003$ & $23: 40: 12$ & $00: 47$ & 7.62 & -15.18 & 2.82 & SW & 0.67 & 0.73 & 93 & 6.8 \\
\hline 24/01/2004 & $02: 32: 22$ & $00: 52$ & 14.17 & 11.96 & -0.62 & SW & 0.63 & 0.55 & 36 & 14.4 \\
\hline
\end{tabular}


Table 1. Continued.

\begin{tabular}{|c|c|c|c|c|c|c|c|c|c|c|}
\hline $\begin{array}{l}\text { Date } \\
\text { DD/MM/YYYY }\end{array}$ & $\begin{array}{l}\text { Time [UT] } \\
\text { HH:MM:SS }\end{array}$ & $\begin{array}{l}\text { Duration [s] } \\
\text { MM:SS }\end{array}$ & $\begin{array}{r}x_{\mathrm{GSE}} \\
R_{\mathrm{E}}\end{array}$ & $\begin{array}{r}y_{\mathrm{GSE}} \\
R_{\mathrm{E}}\end{array}$ & $\begin{array}{r}z_{\mathrm{GSE}} \\
R_{\mathrm{E}}\end{array}$ & $\begin{array}{l}\text { Operational } \\
\text { mode }\end{array}$ & $\Delta B / B$ & $\Delta n / n$ & $\begin{array}{r}V_{\mathrm{A}} \\
\mathrm{km} \mathrm{s}^{-1}\end{array}$ & $M_{\mathrm{A}}$ \\
\hline $24 / 01 / 2004$ & $02: 36: 36$ & $02: 22$ & 14.19 & 11.96 & -0.65 & SW & 0.51 & 0.67 & 36 & 14.4 \\
\hline $04 / 02 / 2004$ & $19: 42: 42$ & $00: 30$ & 14.57 & 8.95 & 1.8 & SW & 0.34 & 0.47 & 105 & 4.9 \\
\hline $16 / 03 / 2004$ & 09:36:06 & $00: 42$ & 17.97 & -3.92 & -0.63 & SW & 0.47 & 0.54 & 80 & 5.2 \\
\hline $16 / 03 / 2004$ & 11:05:07 & $00: 54$ & 18.2 & -4.35 & -1.5 & SW & 0.37 & 0.30 & 98 & 4.2 \\
\hline 23/03/2004 & $07: 32: 10$ & 01:18 & 15.65 & -4.16 & 2.42 & SW & 0.74 & 0.84 & 44 & 8.3 \\
\hline 28/03/2004 & $11: 18: 49$ & $00: 55$ & 16.87 & -8.76 & -3.22 & SW & 0.45 & 0.60 & 155 & 4.5 \\
\hline 28/03/2004 & $21: 40: 38$ & $00: 35$ & 13.75 & -9.77 & -8.27 & SW & 0.60 & 0.75 & 126 & 4.9 \\
\hline 28/03/2004 & $21: 44: 02$ & $00: 46$ & 13.73 & -9.76 & -8.28 & SW & 0.46 & 0.67 & 126 & 4.9 \\
\hline 02/04/2004 & $15: 51: 08$ & 01:05 & 12.72 & -10.84 & -8.42 & SW & 0.58 & 0.60 & 68 & 5.4 \\
\hline $11 / 04 / 2004$ & 08:18:18 & $01: 13$ & 13.55 & -9.12 & 2.24 & SW & 0.60 & 0.57 & 54 & 7.5 \\
\hline $14 / 04 / 2004$ & $00: 53: 33$ & 01:09 & 13.93 & -12.73 & -2.21 & SW & 0.60 & 0.62 & 83 & 4.8 \\
\hline $15 / 04 / 2004$ & $22: 05: 23$ & 01:03 & 10.97 & -7.27 & 4.55 & MS & 0.57 & 0.67 & 74 & 9.4 \\
\hline $19 / 04 / 2004$ & 11:03:19 & $00: 57$ & 7.27 & -12.84 & -9.75 & MS & 0.28 & 0.40 & 53 & 6.6 \\
\hline $19 / 04 / 2004$ & $11: 06: 23$ & $02: 13$ & 7.25 & -12.82 & -9.76 & MS & 0.33 & 0.46 & 53 & 6.6 \\
\hline 21/04/2004 & $16: 03: 46$ & $00: 31$ & 8.82 & -14.86 & -8.34 & SW & 0.67 & 0.81 & 61 & 6.4 \\
\hline $25 / 04 / 2004$ & $20: 18: 14$ & 01:41 & 11.26 & -14.74 & -1.13 & SW & 0.57 & 0.74 & 74 & 5.2 \\
\hline 05/05/2004 & 08:12:09 & $00: 46$ & 8.13 & -16.48 & -2.69 & SW & 0.48 & 0.55 & 65 & 6.7 \\
\hline 05/05/2004 & $08: 17: 34$ & 01:04 & 8.11 & -16.5 & -2.74 & SW & 0.38 & 0.40 & 64 & 6.8 \\
\hline 05/02/2005 & $09: 45: 14$ & 01:00 & 13.92 & 3.16 & -9.82 & SW & 0.35 & 0.62 & 48 & 7.5 \\
\hline $19 / 03 / 2005$ & $11: 24: 19$ & 01:03 & 17.9 & -4.53 & -1.57 & SW & 0.26 & 0.34 & 78 & 4.9 \\
\hline $19 / 03 / 2005$ & $14: 50: 55$ & $01: 12$ & 18.03 & -5.5 & -3.59 & SW & 0.37 & 0.44 & 60 & 6.0 \\
\hline 26/03/2005 & $23: 44: 16$ & $00: 58$ & 16 & -8.74 & -6.66 & SW & 0.48 & 0.60 & 77 & 7.9 \\
\hline $17 / 04 / 2005$ & $01: 26: 20$ & $00: 53$ & 13.59 & 12.84 & -2.4 & SW & 0.50 & 0.47 & 50 & 8.2 \\
\hline $27 / 04 / 2005$ & 03:54:39 & $02: 53$ & 6.82 & -14.38 & -9.39 & SW & 0.66 & 0.71 & 41 & 9.1 \\
\hline 23/01/2006 & $03: 32: 44$ & $00: 57$ & 6.79 & 11.1 & 3.58 & MS & 0.46 & 0.41 & 103 & 3.6 \\
\hline 24/01/2006 & $05: 43: 43$ & $01: 31$ & 13.21 & 7.12 & -9.98 & SW & 0.58 & 0.67 & 48 & 11.6 \\
\hline 24/01/2006 & 09:39:27 & 01:00 & 11.67 & 4.65 & -10.8 & SW & 0.35 & 0.38 & 72 & 7.3 \\
\hline 26/01/2006 & 08:36:02 & $00: 42$ & 14.68 & 9.71 & -7.6 & SW & 0.28 & 0.35 & 77 & 6.1 \\
\hline 26/01/2006 & 09:00:39 & $01: 33$ & 14.65 & 9.54 & -7.79 & SW & 0.24 & 0.29 & 84 & 5.8 \\
\hline 01/03/2006 & $04: 48: 23$ & 01:10 & 10.04 & -3.83 & -11 & MS & 0.47 & 0.44 & 85 & 4.3 \\
\hline 02/03/2006 & $19: 41: 25$ & $01: 37$ & 18.46 & 0.9 & -5.01 & SW & 0.42 & 0.40 & 44 & 8.2 \\
\hline 03/03/2006 & 12:05:01 & 01:07 & 11.5 & -4.07 & -11 & SW & 0.54 & 0.48 & 45 & 8.7 \\
\hline 29/03/2006 & $10: 51: 05$ & 02:06 & 12.68 & -9.02 & -9.97 & SW & 0.41 & 0.53 & 45 & 7.2 \\
\hline 29/03/2006 & $11: 58: 52$ & $00: 56$ & 11.95 & -9.01 & -10.2 & SW & 0.53 & 0.49 & 43 & 7.6 \\
\hline 26/04/2006 & 09:17:08 & $01: 14$ & 12.3 & -3.12 & -13.5 & SW & 0.58 & 0.66 & 36 & 7.8 \\
\hline 26/04/2006 & $18: 53: 11$ & 01:44 & 9.59 & -8.1 & -14.82 & SW & 0.57 & 0.48 & 32 & 10.3 \\
\hline 26/04/2006 & $19: 49: 43$ & 00:59 & 9.17 & -8.5 & -14.75 & SW & 0.62 & 0.56 & 32 & 9.9 \\
\hline 28/04/2006 & $23: 02: 12$ & 01:03 & 10.75 & -5.72 & -15 & SW & 0.35 & 0.50 & 67 & 5.2 \\
\hline 29/04/2006 & $04: 51: 46$ & 00:40 & 8.61 & -8.48 & -15.12 & SW & 0.29 & 0.50 & 55 & 6.7 \\
\hline
\end{tabular}

by rims of enhanced $B$ and $n$. The maximum values of $B$ in the upstream and downstream rims are $4.8 \mathrm{nT}$ and $5.7 \mathrm{nT}$ (26\% and $50 \%$ increase with respect to the average ambient value). The corresponding maximum values of plasma density are $6.3 \mathrm{~cm}^{-3}$ and $7.6 \mathrm{~cm}^{-3}$ (23\% and $49 \%$ increase). The solar wind thermal pressure (panel d) shows a similar depression as $B$ and $n$. This is due to the fact that the SW temperature shows no variation during the presented time interval. Total velocity $(e)$ diminishes from $\sim 340 \mathrm{~km} \mathrm{~s}^{-1}$ to $\sim 300 \mathrm{~km} \mathrm{~s}^{-1}$ (by 13\%) at the upstream edge of the caviton, and shows a peak of $365 \mathrm{~km} \mathrm{~s}^{-1}$ (7\% increase) inside the caviton. There are velocity fluctuations in the surrounding medium (at 09:11:52 UT and 09:13:58 UT) that reach similar or even lower values than the one described here. Hence it is not clear if the velocity fluctuation observed at the time of the caviton was really caused by it. The energy HS spectrogram $(g)$ shows an intense suprathermal ion population inside as well as outside of the caviton, while the LS energy spectrogram $(h)$ shows a continuous solar wind beam centered at $\sim 600 \mathrm{eV}$. The HS spectrogram and the highly disturbed $B$ and $n$ panels show that the caviton is located well inside the foreshock.

In Fig. 3, we show cuts of the ion distribution functions from the "high-G" section inside the caviton at 


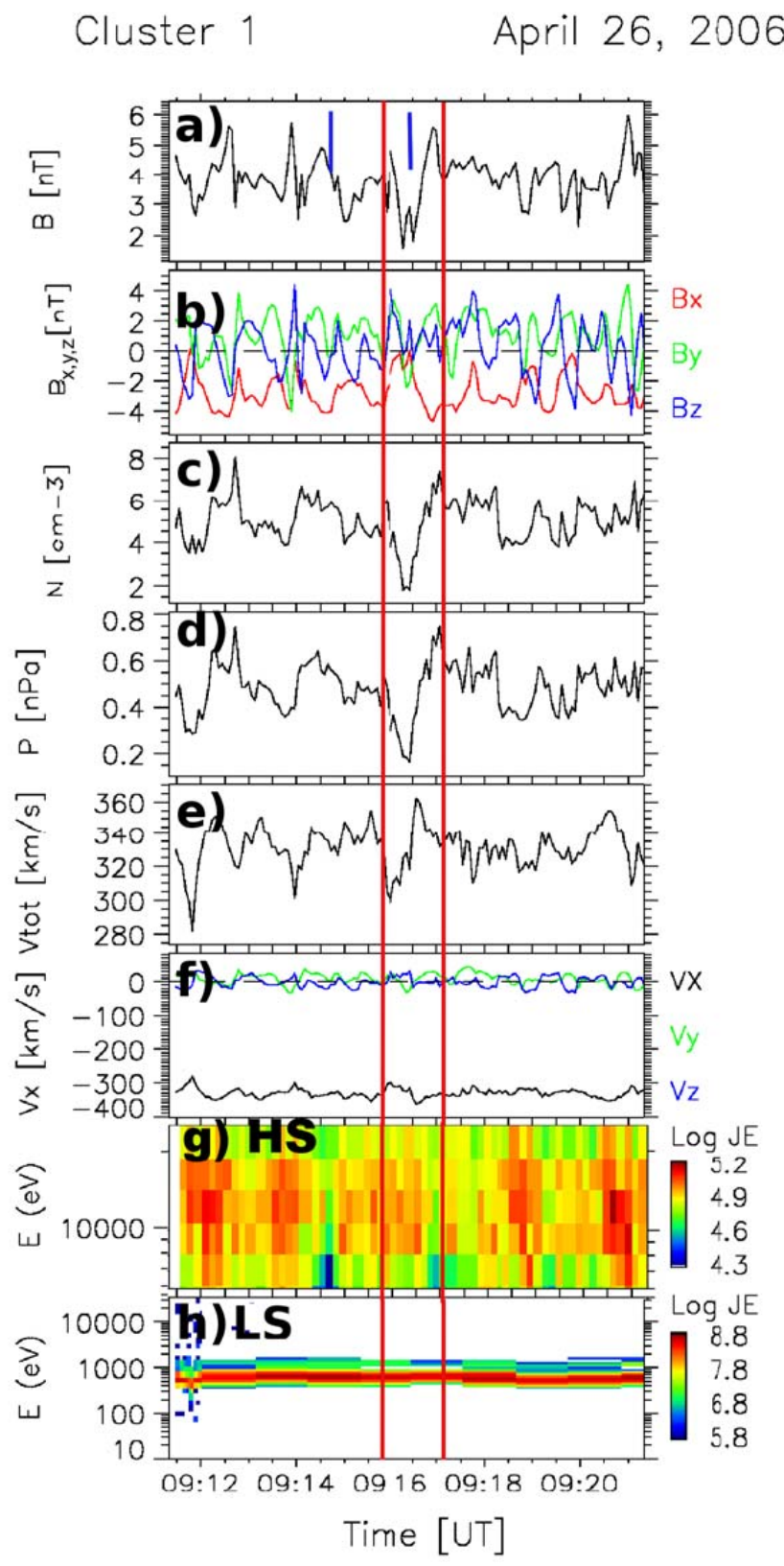

Fig. 2. Foreshock caviton observed on 26 April 2006. The two red vertical lines delimit the time of the caviton. The two small blue vertical lines mark the times of ion distributions shown in Fig. 3.

09:16:28.759 UT (top panels) and those in the surrounding region at 09:15:01.839 UT (bottom panels). The times of the distribution are marked in Fig. 2 with two short vertical blue lines. The panels show the logarithm of phase space density in the spacecraft frame of reference. Since the spacecraft was operating in a solar wind mode and the data is provided by the HS section, the SW beam does not appear in these panels. $v_{\text {par }}$ and $v_{\text {perp }}$ stand for velocities parallel and perpendicular to the magnetic field. We can see that for both times, a hot, diffuse ion distribution is present. Hence, ion distributions

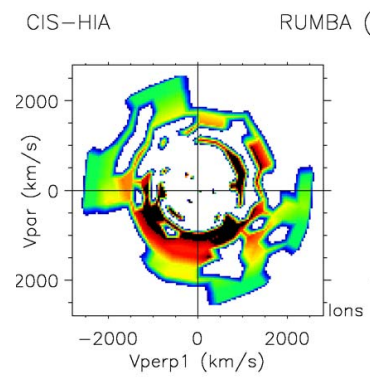

UMBA (SC 1) 26/Apr/2006 09:15:01.839
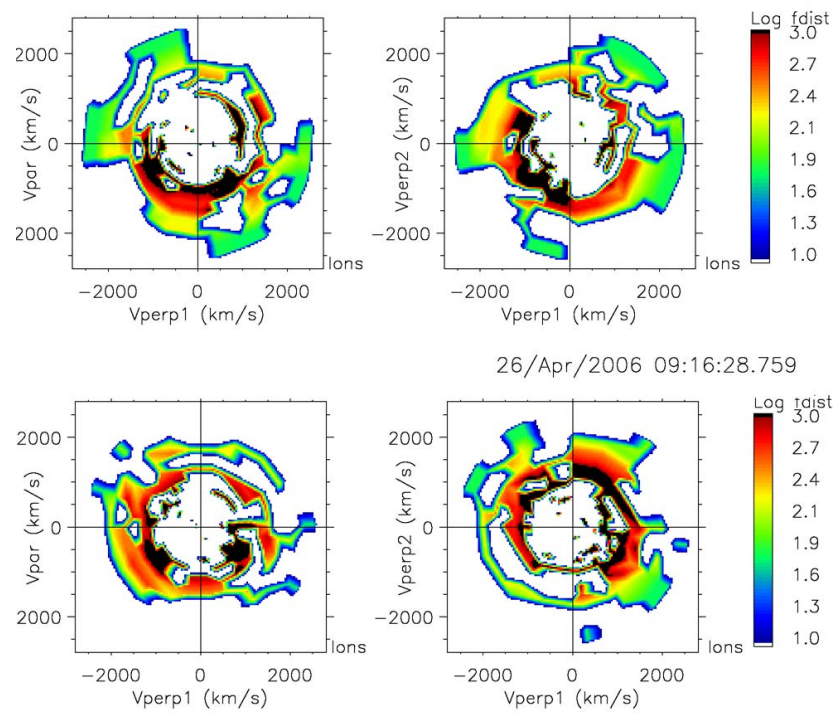

26/Apr/2006 09:16:28.759

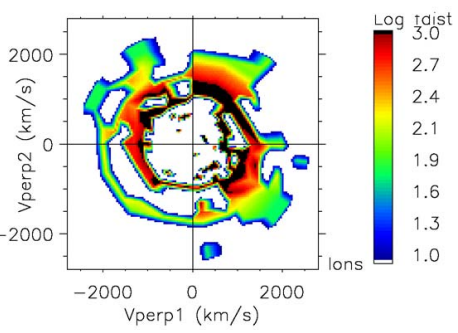

Fig. 3. Ion distributions just outside (top) and inside (bottom) the caviton. The times of the distributions are marked in Fig. 2 with two short vertical blue lines. Logarithm of ion distribution function is shown. The distributions are obtained from the "high-G" section of the CIS-HIA instrument, so the solar wind distribution does not appear on these panels. $v_{\text {par }}$ and $v_{\text {perp }}$ refer to velocities parallel and perpendicular to the IMF.

inside the caviton and in its surroundings are of the same type. We also revised the ion distributions throughout the event and found that they remain very similar.

\subsection{Surrounding ULF waves}

In this section, we show how the observational properties of foreshock cavitons differ from those of the surrounding ULF waves.

Panels a through $d$ in Fig. 4 present Cluster 1 observations between 08:59:57 UT and 09:21:04 UT on 26 April 2006. $\boldsymbol{B}$ field magnitude is exhibited on panel a, $B_{x}$ component in panel b, $B_{y}$ (thick, black line) and $B_{z}$ (thin, blue line) components in panel $\mathrm{c}$ and the plasma density in panel $\mathrm{d}$. During this time, the IMF and SW density show no large variations other than those caused by the ULF waves and the caviton, so meaningful averages of both quantities (3.76 nT and $5.14 \mathrm{~cm}^{-3}$ ) could be calculated.

We first perform fast Fourier and minimum variance analyses (MVA) of the ULF waves during the exhibited time interval. The power spectrum (Fig. 4f) shows that these waves are predominantly transverse although a strong compressive component is also present. The spectrum of transverse component peaks for periods $\sim 50 \mathrm{~s}$. The MVA analysis (Fig. $4 \mathrm{~g}$ ) reveals that the ratio of intermediate to minimum variance is only 2.3 , which introduces a large uncertainty in their direction of propagation with respect to the local $\boldsymbol{B}$ field 

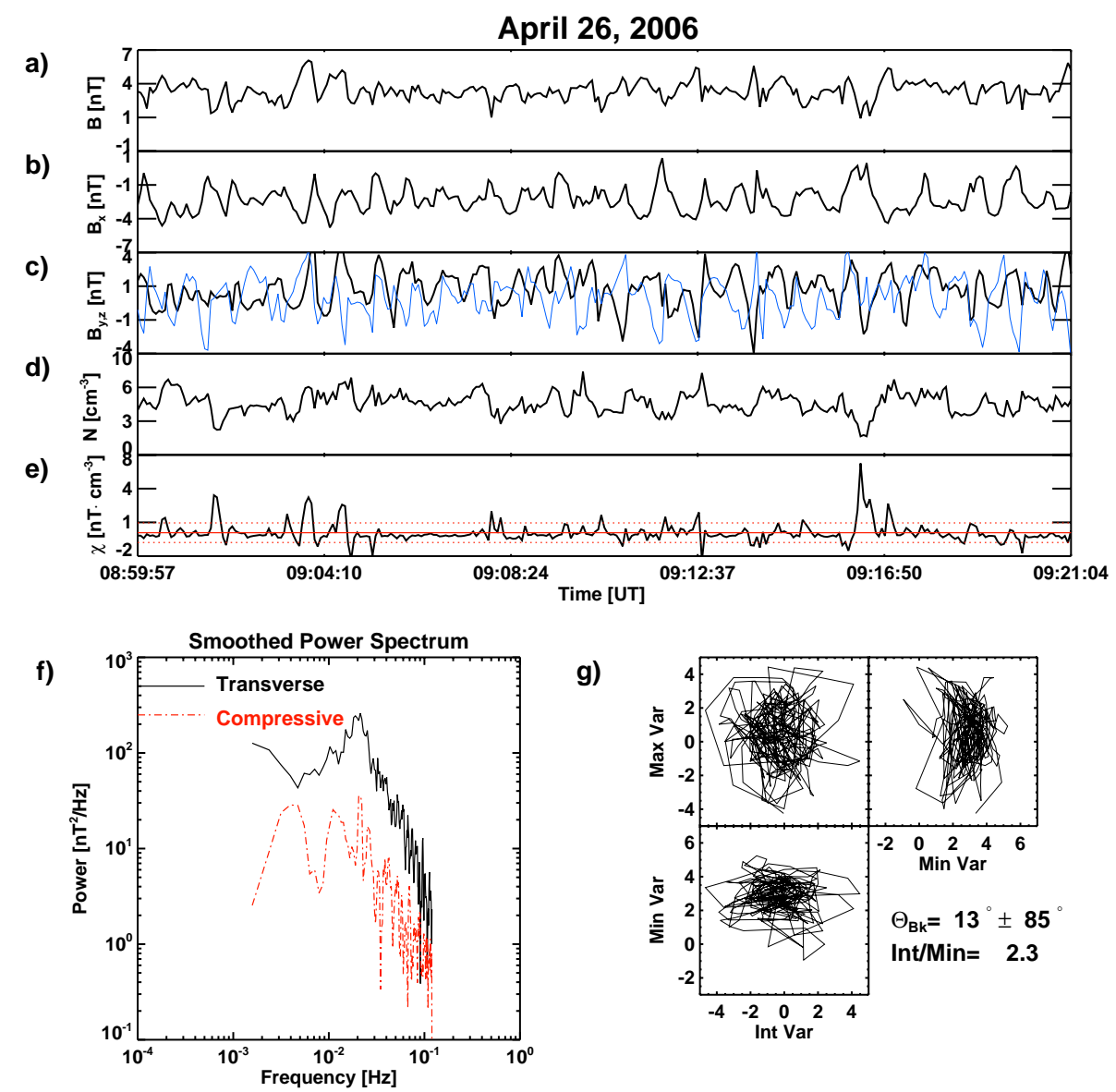

g)

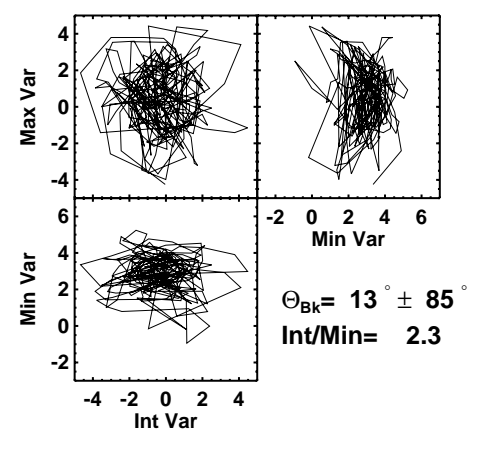

Fig. 4. (a) magnetic field magnitude, (b) $x_{\mathrm{GSE}}$ component and (c) $y_{\mathrm{GSE}}$ and $z_{\mathrm{GSE}}$ components of $\boldsymbol{B}$ field, (d) plasma density and (e) the $\chi$ function between 08:59:57 UT and 09:21:04 UT on 26 April 2006. (f) power spectrum of ULF waves. (g) minimum variance analysis results.

direction $\left(\theta_{\mathrm{Bk}}=13^{\circ} \pm 85^{\circ}\right)$. This error was estimated according to Hoppe et al. (1981).

During the presented time interval, we identified 27 wavefronts in the $x$ component of the magnetic field and 27 in density (see Fig. 4a and d) of different amplitudes and durations. However, the $\boldsymbol{B}$ field magnitude does not seem to correlate so well with the density. There are 41 peaks, which have smaller amplitudes. In the following paragraphs we compare perturbations in $B$ and $n$ caused by the ULF waves and the foreshock cavitons.

Figure 5 shows distributions of (panel a) relative amplitudes $(\Delta B / B)$ of ULF fluctuations in $\boldsymbol{B}$ field, (panel b) relative density amplitudes $(\Delta n / n)$ of ULF waves, and (panel c) wave durations in $B$ and (panel d) in $n$. In Fig. 5a and $\mathrm{b}$ the red columns represent the amplitudes of wave minima while the blue bars represent the amplitudes of the wave maxima. Relative frequencies are shown in each panel. The black column represents the foreshock caviton. The average, the median values and the standard deviations of the distributions are also given. In the case of $\Delta B / B$ and $\Delta n / n$, these values were calculated from the sample that includes the wave maxima and the absolute values of their minima. We can see that the average (median) $\boldsymbol{B}$ field depletions of surrounding ULF waves were $0.19(0.20) \pm 0.15$ and average (median) depletions in density were $0.21(0.20) \pm 0.18$. This is about three times less than the corresponding depletions produced by the caviton. Also, the average (median) periods of waves in $\boldsymbol{B}$ field and $n$ were $29(25) \mathrm{s} \pm 21 \mathrm{~s}$ and $50(37) \mathrm{s} \pm 33 \mathrm{~s}$, respectively. Again, with the duration of $76 \mathrm{~s}$, the caviton lasts longer than this.

Although the caviton produced the largest negative depletions in $B$ and $n$ during the studied time interval, there were still a few ULF fluctuations that were almost as deep. Hence, the described statistics are not enough to distinguish cavitons from the surrounding ULF waves. However, cavitons produce $B$ and $n$ depletions simultaneously. Their shapes in $B$ and $n$ data are very similar. In order to show this, we define the following function:

$\chi(t)=(n(t)-<n>) \cdot(B(t)-<B>)$,

where $<n>$ and $<B>$ are the average values calculated on the exhibited time interval. We plot this function in Fig. 4e. We see that the caviton produces by far the largest positive peak with the value of 7.2. The average value of $\chi$ during the 

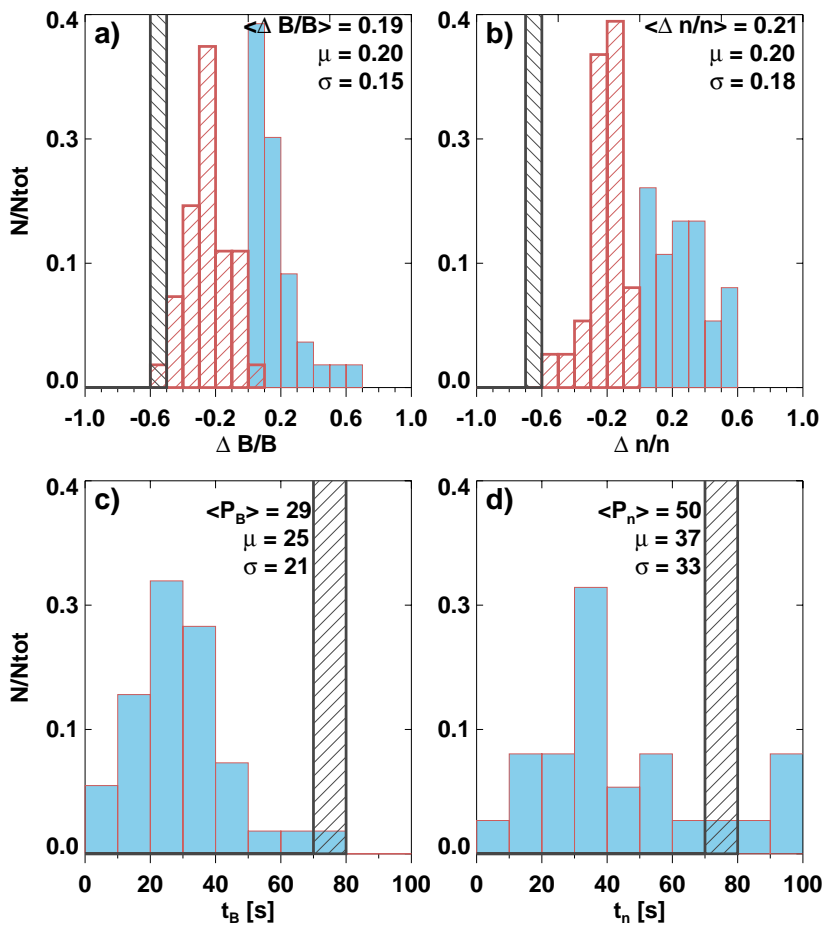

Fig. 5. Distributions of (a) relative amplitudes $(\Delta B / B)$ of ULF fluctuations, (b) relative density amplitudes $(\Delta n / n)$ of ULF waves, (c) wave durations in $\boldsymbol{B}$ field and (d) wave durations in $n$. The red columns in (a) and (b) represent the amplitudes of wave minima while the blue bars represent the amplitudes of the wave maxima. The black column represents the foreshock caviton.

presented time interval (the covariance between $B$ and $n$, the thick red line in Fig. 4e) is $\langle\chi\rangle=0.19$ and the standard deviation of $\chi$ (the dashed red lines in Fig. 4e) is $\sigma_{\chi}=0.77$. This means that the peak produced by the caviton is $9 \sigma_{\chi}$ larger than $\langle\chi\rangle$. The largest value of $\chi$ produced by the ULF fluctuations is 4 , which is slightly less than $5 \sigma_{\chi}$ larger than $<\chi>$.

Hence we add an additional requirement for an event to be recognized as a foreshock caviton: the value of $\chi$ during the event must reach values at least 5 standard deviations $\sigma_{\chi}$ larger than the average value of $\langle\chi\rangle$ during the studied time interval.

Finally, we calculate the Pearson correlation coefficients for $\boldsymbol{B}$ field and density for subintervals with and without the caviton. Their respective values are 0.77 and 0.38 , which again shows the high correlation of $B$ and $n$ inside the cavitons.

\subsection{Statistical analysis}

We surveyed the Cluster 1 data between the years 20012006. We found 92 foreshock cavitons, of which 79 were observed when the CIS was in the SW operational mode and 13 were observed when it was in the MS operational mode. We should stress out that the relatively small number of events in our sample is a consequence of our stringent selection criteria and the fact that the Cluster 1 spacecraft spent in total only $\sim 50$ days in the foreshock region during the mentioned time period. It is likely that their formation processes are very common in the regions populated by compressive ULF waves and that the cavitons are much more recurrent. Hence, the statistics presented in this section apply only for those foreshock cavitons that evolved sufficiently in order to be distinguished from the surrounding ULF fluctuations.

Figure 6 shows the positions of these events in the GSE coordinate system. The crosses mark the locations of the cavitons observed when spacecraft was operating in the SW modes and the diamonds mark those events that were observed in the MS modes. The diamonds tend to appear closer to the bow shock than the crosses. This is because the MS modes are used during time intervals between $2 \mathrm{~h}$ before the inbound crossing of a nominal bow shock, until $2 \mathrm{~h}$ after its outbound crossing, so when the spacecraft is closer to the shock. In the GSE coordinate system, the Earth is in the center, the $x$ axis points towards the Sun, the $y$ axis is in the ecliptic plane pointing towards dusk (opposite the Earth's motion) and the $z$ axis is parallel to the ecliptic pole. Panels a, $\mathrm{b}$ and $\mathrm{c}$ show $x_{\mathrm{GSE}} y_{\mathrm{GSE}}, x_{\mathrm{GSE}} z_{\mathrm{GSE}}$ and $z_{\mathrm{GSE}} y_{\mathrm{GSE}}$ planes, respectively. The dashed curves on top panels in Fig. 6 represent the nominal bow shock as modeled by Narita et al. (2004). We can see that the caviton locations in Fig. 6 extend more dawnward $\left(y_{\mathrm{GSE}} \gtrsim-18 R_{\mathrm{E}}\right)$ than they do duskward $\left(y_{\mathrm{GSE}} \lesssim 15 R_{\mathrm{E}}\right)$. $R_{\mathrm{E}}$ stands for Earth radius. Also, the range of negative $z_{\mathrm{GSE}}$ values $\left(\gtrsim-18 R_{\mathrm{E}}\right)$ is larger than the range of positive $z_{\mathrm{GSE}}$ values $\left(\lesssim 8 R_{\mathrm{E}}\right)$. There seems to be a region $\left(-10 R_{\mathrm{E}} \leq z \leq 0 R_{\mathrm{E}}\right.$ and $\left.10 R_{\mathrm{E}} \leq x \leq 17 R_{\mathrm{E}}\right)$ where no cavitons were observed.

The locations at which the foreshock cavitons were detected are influenced by the caviton's actual distribution in the Earth's foreshock and by the way the spacecraft traveled through the foreshock. At the beginning of the Cluster mission, including the period between 2001 and 2006, the Cluster spacecraft were in a highly elliptical orbit that was almost perpendicular to the ecliptic. Whenever the spacecraft crossed the ecliptic, their $x_{\mathrm{GSE}}$ coordinates were large, while at small $x_{\mathrm{GSE}}$ the $z_{\mathrm{GSE}}$ was large. Events with small $x_{\mathrm{GSE}}$ and small $z_{\mathrm{GSE}}$ coordinates could still be observed when the angle between the IMF and the radial direction was large. As we can see in Fig. 6 there is one such event at $x_{\mathrm{GSE}} \sim 7 R_{\mathrm{E}}$ and $z_{\mathrm{GSE}} \sim-2 R_{\mathrm{E}}$.

From Fig. 6 we also see that the cavitons were predominantly observed upstream of the dawn-side bow shock. This can be explained in terms of the orientation of the nominal Parker spiral. Reflected particles, responsible for foreshock formation, stream along the IMF lines, and it is due to this that the orientation of the foreshock is preferentially in the negative $y_{\mathrm{GSE}}$ direction. 

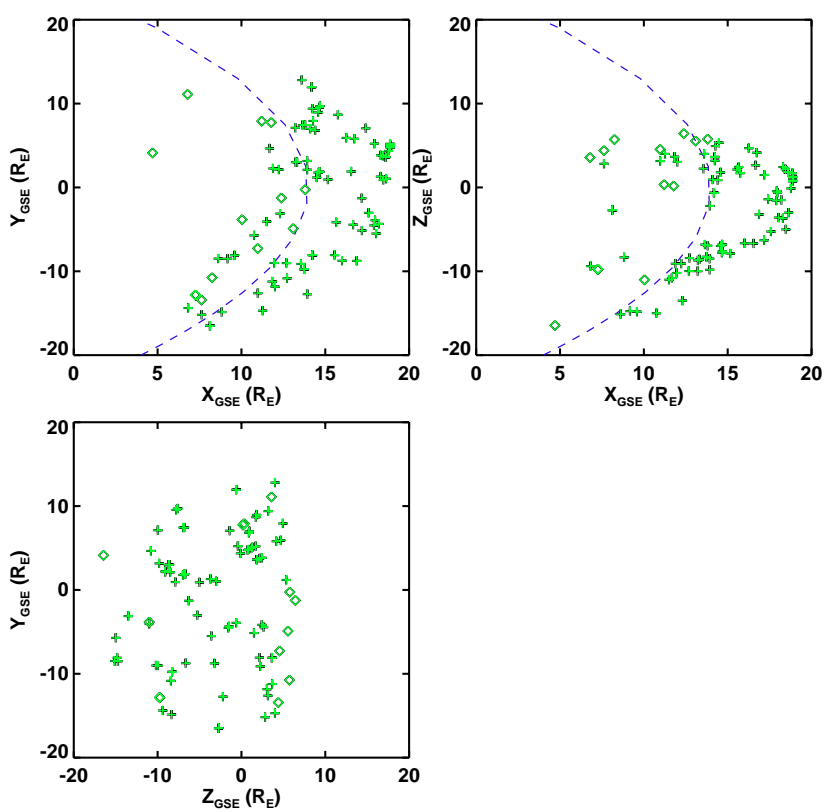

Fig. 6. Coordinates of the observed foreshock cavitons in the GSE coordinate system. The crosses represent the events observed in the SW modes, while the diamonds represent those cavitons observed in the MS modes. The dashed curve on the two top panels represents the nominal bow shock. A nominal bow shock model of Narita et al. (2004) was used.

\subsubsection{Ambient solar wind and IMF conditions}

In this section we analyze properties of SW and IMF at times when the cavitons were observed and compare them to the overall SW and IMF properties during the period 2001-2006.

The top row in Fig. 7 shows the statistics of properties of the ambient SW and IMF during times when the cavitons were observed. The presented quantities are averages calculated on intervals of several minutes around the observed cavitons. On vertical axes we show relative frequency $\left(N / N_{\text {tot }}\right)$. The columns show from left to right: magnetic field magnitude in units of nanoteslas (nT), SW plasma density $\left(\mathrm{cm}^{-3}\right)$, velocity $\left(\mathrm{km} \mathrm{s}^{-1}\right)$, Alfvén velocity $\left(\mathrm{km} \mathrm{s}^{-1}\right)$ and Alfvénic Mach number $\left(M_{\mathrm{A}}\right)$. The middle row shows the same statistics but calculated for $7501 \mathrm{~h}$ time intervals during the years 2001-2006, when Cluster 1 was in pristine solar wind. With this we learn about the SW and IMF properties during the mentioned time period and can then compare them to the properties at times when the cavitons were observed (shown in the top row). We obtained the distributions in the bottom row by dividing the distributions from the top row by the distributions form the middle row, bin by bin.

From Fig. 7 we can see that the cavitons appear for a wide range of IMF magnitudes ( $3 \mathrm{nT}<B<12 \mathrm{nT}$ ). The average and the median $B$ magnitude in the sample are $6.9 \mathrm{nT}$ and $6.6 \mathrm{nT}$, respectively. The cavitons also appear for almost any SW density (between $\lesssim 2 \mathrm{~cm}^{-3}$ and $20 \mathrm{~cm}^{-3}$ ), velocity $\left(200 \mathrm{~km} \mathrm{~s}^{-1}<V<700 \mathrm{~km} \mathrm{~s}^{-1}\right)$ and Alfvénic Mach number $\left(2<M_{\mathrm{A}}<15\right)$. The average density, velocity and $M_{\mathrm{A}}$ are $4.8 \mathrm{~cm}^{-3}, 456 \mathrm{~km} \mathrm{~s}^{-1}$ and 6.5 , respectively. Their respective median values are very similar: $4.5 \mathrm{~cm}^{-3}, 445 \mathrm{~km} \mathrm{~s}^{-1}$ and 6.0 .

We can see that the average and median properties of the SW and IMF, shown in the middle row, differ from those in the top row. During the period 2001-2006, the average (median) IMF strength was $6.0 \mathrm{nT}(5.8 \mathrm{nT})$. The average (median) SW density, velocity, Alfvén velocity and Alfvénic Mach number were $7.8 \mathrm{~cm}^{-3}\left(6.5 \mathrm{~cm}^{-3}\right)$, $424 \mathrm{~km} \mathrm{~s}^{-1}\left(399 \mathrm{~km} \mathrm{~s}^{-1}\right), 75 \mathrm{~km} \mathrm{~s}^{-1}\left(78 \mathrm{~km} \mathrm{~s}^{-1}\right)$ and 6.5 (6.0), respectively.

From Fig. 7 we can see that proportionally more cavitons were found for higher-than-average IMF strengths, while most of the cavitons were observed during times of lowerthan-average solar wind densities. Very few foreshock cavitons were found for Alfvén Mach numbers larger than 10.

The bottom row in Fig. 7 shows these tendencies. If the cavitons were observed with equal probability for all IMF and SW conditions, the histograms in this row would be flat. However, this is not the case. It can clearly be seen that cavitons favor higher $\boldsymbol{B}$ fields, lower densities, larger velocities and Alfvén speeds, when compared to the average SW properties.

\subsubsection{Caviton properties}

Figure 8 shows the statistics of foreshock caviton properties. The panels show the distributions according to panel a the relative depth of the depression of the magnetic field magnitude, $\Delta B / B$, panel $\mathrm{b}$ the relative depth of the depression of plasma density, $\Delta n / n$, panel c the caviton duration in the data and panel d the calculated extents (in $R_{\mathrm{E}}$ ). The latter were calculated by multiplying the caviton durations with the average surrounding SW speed.

Three distributions are shown on Fig. 8: the dark-blue color represents the statistics for the cavitons that were observed in MS operational mode, the light-blue shows the distributions of the cavitons observed in solar wind mode and the white columns with red borders show the statistics for the entire sample.

We see that the $\Delta B / B$ values range between 0.2 and 0.9 with the average and the median values being 0.47 . The distribution decreases strongly beyond the values of 0.7 .

The picture is similar for $\Delta n / n$ values. These values also range between 0.2 and 0.9 . The distribution looks more symmetric around the average and median values of 0.5 and 0.52 , respectively. The distribution is quite flat between 0.3 and 0.7 .

The caviton durations in the data range between $20 \mathrm{~s}$ and $180 \mathrm{~s}$. The average and the median durations are $65.4 \mathrm{~s}$ and $58 \mathrm{~s}$ and the distribution shows a large spread of $29.3 \mathrm{~s}$. The most common durations are between $50 \mathrm{~s}$ and $70 \mathrm{~s} \mathrm{(41} \mathrm{cavi-}$ tons, $44.6 \%$ of the sample). 


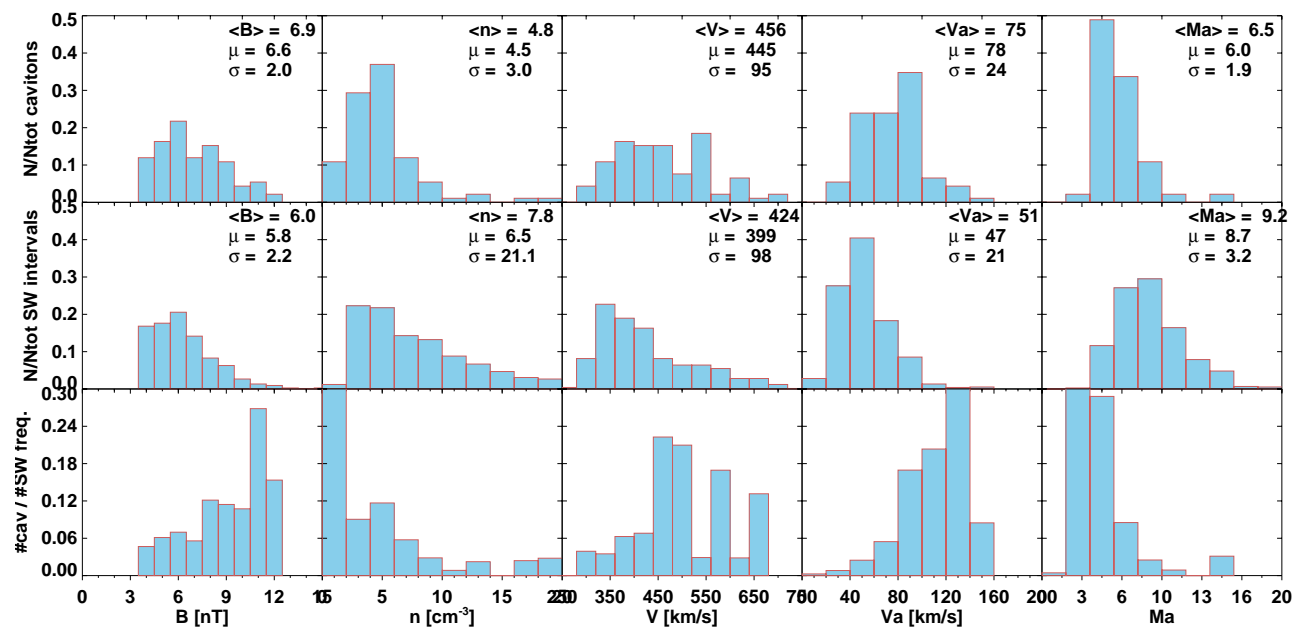

Fig. 7. Top row: properties of the solar wind and IMF at times when the cavitons were observed. Middle row: solar wind and IMF properties for the period between 2001-2006. Bottom row: normalized statistics of the SW and IMF properties at times of caviton observations. The columns show, from left to right: magnetic field magnitude (nT), plasma density $\left(\mathrm{cm}^{-3}\right)$, SW velocity $\left(\mathrm{km} \mathrm{s}^{-1}\right)$, Alfvén velocity in the SW $\left(\mathrm{km} \mathrm{s}^{-1}\right)$ and Alfvénic Mach number $\left(M_{\mathrm{A}}\right)$.

The extents range between 1 and $13 R_{\mathrm{E}}$ with the average and median values being $4.6 R_{\mathrm{E}}$ and $4.1 R_{\mathrm{E}}$, respectively. The most common extents are between $3 R_{\mathrm{E}}$ and $5 R_{\mathrm{E}}(56$ cavitons, $61 \%)$.

We have looked at possible correlations between different caviton properties. The only meaningful correlation was found between $\Delta B / B$ and $\Delta n / n$ (Fig. 9). Figure 9 shows that the two quantities are very well correlated with the linear Pearson correlation coefficient being 0.85 . In the figure, the asterisks represent the cavitons observed in the SW operational mode and the diamonds illustrate those observed on the MS mode. There does not seem to be any difference between the two subgroups, as expected. The thick line is a linear fit to the distribution. The strong $\Delta B / B$ vs. $\Delta n / n$ correlation points towards the fast magnetosonic nature of foreshock cavitons.

\subsubsection{Cavitons in the solar foreshock coordinate system}

Solar foreshock coordinate system relates upstream coordinates to a normalized bow shock. It enables us to compare the locations of upstream phenomena by eliminating the effects of variable solar wind properties and IMF orientation.

Solar foreshock coordinates (SFC) were first introduced by Greenstadt and Baum (1986) who studied the location of the ULF compressional waves in the Earth's foreshock. Meziane and D'Uston (1998) used these coordinates in order to describe the observed locations of the intermediate ion boundary and then compared their observations to those by Greenstadt and Baum (1986).

Foreshock cavitons are always located upstream of the quasi-parallel bow shock. They are surrounded by intense compressive ULF waves and hot suprathermal populations.
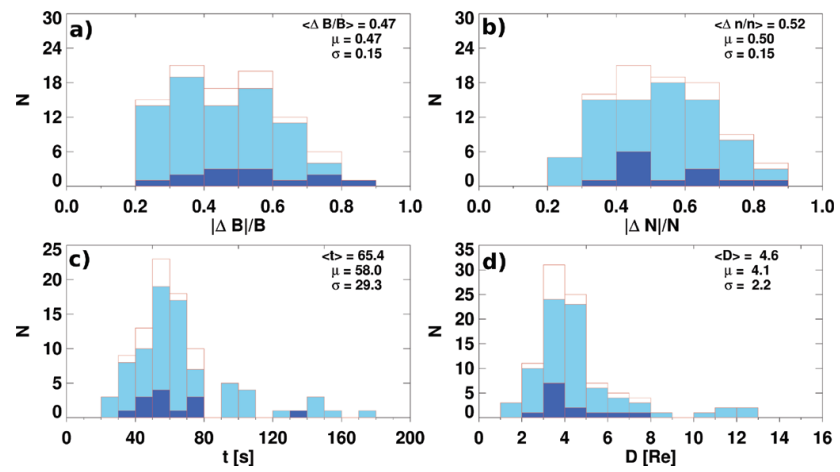

Fig. 8. Caviton properties. The following quantities are shown: (a) the relative depth of the magnetic field magnitude depression, $\Delta B / B$; (b) the relative depth of the plasma-density depression, $\Delta n / n$; (c) duration in the data and (d) extent of the cavitons in units of $R_{\mathrm{E}}$. The dark-blue columns show distributions for cavitons observed in MS modes, the light-blue for those observed in solar wind modes while white columns show distributions for the entire sample.

They should therefore always appear downstream of the ULF compressional boundary and of the intermediate ion boundary. We show this by comparing the SFC of foreshock cavitons with those of the two boundaries.

In order to calculate these coordinates one has to first calculate the cross section of a model bow shock with the $\boldsymbol{B}-\boldsymbol{x}$ plane, which is defined by the observation point, the $x$ axis and the IMF direction. The locations of all points on this plane are described by rectangular $(x, \eta)$ coordinates (see Fig. 10). The locations of the foreshock phenomena are described by another set of coordinates, $X_{\mathrm{F}}$ and $D_{\mathrm{BT}}$. $X_{\mathrm{F}}$ is parallel to the $x$ axis and measures the distance between the 


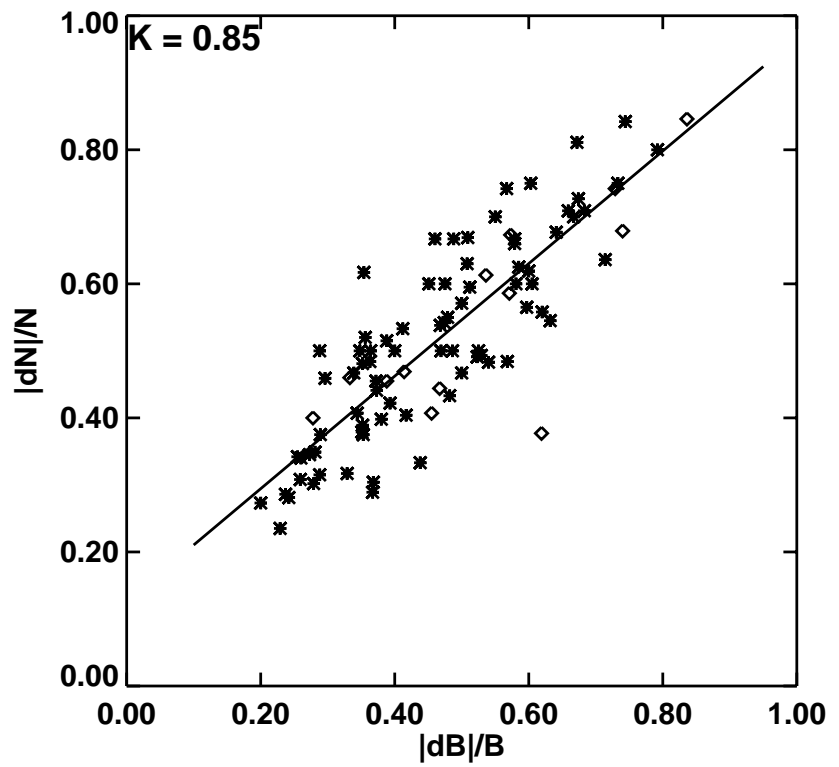

Fig. 9. Correlation between the magnetic field magnitude and plasma-density depletions inside the foreshock cavitons. Asterisks mark cavitons observed in solar wind operational modes while diamonds mark those events that were observed in MS modes.

observation point to the field line which is tangent to the shock. $D_{\mathrm{BT}}$ measures the distance along the tangent field line from the shock to the point on the plane that has the same value of $\eta$ as the observed event.

We follow the procedure described by Greenstadt and Baum (1986). These authors describe the shape of the bow shock by a hyperboloid. Its intersection with the $\boldsymbol{B}-\boldsymbol{x}$ plane is then given by the equation:

$\eta^{2}=A\left(\left(X-B D_{0}\right)^{2}+C D^{2}\right)-D_{\mathrm{BX}}^{2}$,

where $A, B$ and $C$ are constants and their values are 0.04 , 39.22 and 1461 , respectively. $D_{0}=13.5 R_{\mathrm{E}}$ is the geocentric distance of the subsolar point and $D_{\mathrm{BX}}$ is the distance between the Sun-Earth axis and the $(x, \eta)$ plane. In order to obtain the direction of the magnetic field at the time of the cavitons, we average the surrounding $\boldsymbol{B}$ field during time intervals with typical durations of several minutes.

Figure 11 shows positions of the 92 foreshock cavitons from the sample in the SFC. Here we remind the reader that the SFC are calculated from the position of the nominal bow shock model, which is a long-term average. The actual position of the bow shock is time depended and this is why there are some negative $X_{\mathrm{F}}$ values in the figure. The cavitons are represented by different symbols that stand for different cone angles (the angle between the solar wind velocity and the upstream IMF) $\theta_{\mathrm{BV}}$. The asterisks, diamonds, triangles, squares and crosses mark cavitons observed for cone angles between $10^{\circ}$ and $20^{\circ}, 20^{\circ}$ and $30^{\circ}, 30^{\circ}$ and $40^{\circ}, 40^{\circ}$ and $50^{\circ}$ and $50^{\circ}$ and $60^{\circ}$, respectively. The black line is a linear fit to the entire sample. For comparison we also plot the fits for
Solar foreshock coordinates

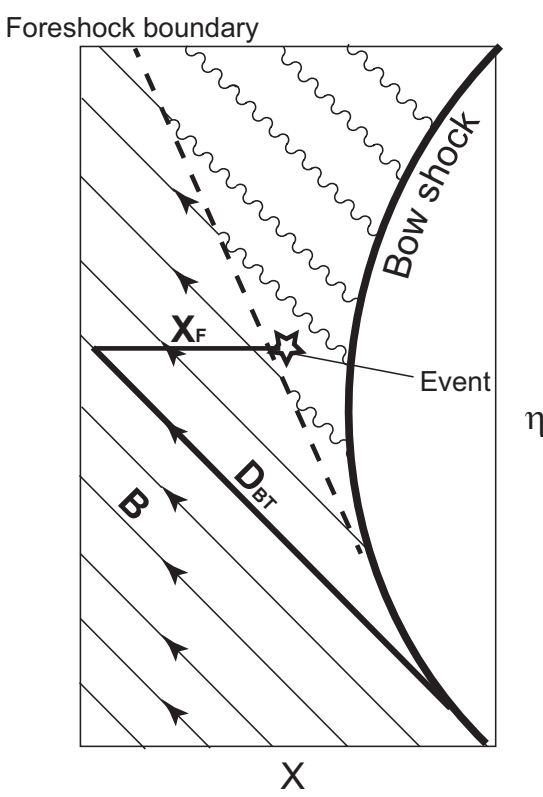

Fig. 10. Solar foreshock coordinate system. The plot was made following Greenstadt and Baum (1986). The definitions of all variables are provided in the text.

the intermediate ion boundary (Meziane and D'Uston, 1998) and the ULF wave boundary (Greenstadt and Baum, 1986) which are represented by a red dash-dotted and a blue dashed lines. The two lines are fits for cone angles between $40^{\circ}$ and $50^{\circ}$. The space in this figure is divided into the foreshock region (left of the two boundaries) and the pristine solar wind (right of the boundaries). The horizontal black dash-dotted line shows the location of the nominal tangent line. The fits for the ULF wave boundary and the intermediate ion boundary match quite well and probably represent the same boundary. The line representing the fit to the cavitons lies further downstream and diverges from the other two boundaries.

We see that foreshock cavitons show larger dispersion around the thick black line for smaller cone angles. This probably has to do with the orbits of the Cluster spacecraft. We can see from the figure that smaller cone angles mean smaller distances from the shock $\left(D_{\mathrm{BT}}\right)$. It seems that at larger $D_{\mathrm{BT}}$ (larger cone angles) the spacecraft could only barely enter the region populated by the cavitons and could therefore survey only a small range of $X_{\mathrm{F}}$. When the cone angle was small, the range of coordinates $X_{\mathrm{F}}$ that the spacecraft could survey was larger, hence the larger dispersion.

In Fig. 12, we plot the caviton SFCs for cone angles between $10^{\circ}$ and $20^{\circ}$ (panel a), $20^{\circ}$ and $30^{\circ}$ (panel b), $30^{\circ}$ and $40^{\circ}$ (panel c), and $40^{\circ}$ and $50^{\circ}$ (panel d). In these panels the thick black line is the same as in Fig. 11, while the black dashed line is a fit to the cavitons shown on each panel. The ULF wave and the intermediate ion boundaries 


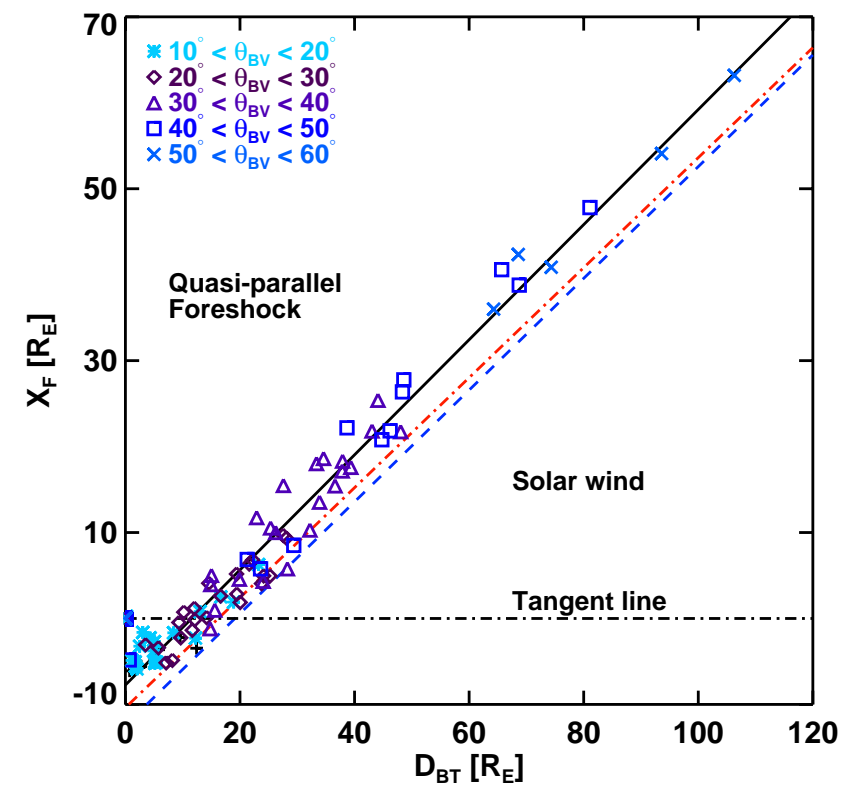

Fig. 11. Positions of foreshock cavitons in solar foreshock coordinates. The $X_{\mathrm{F}}$ and $D_{\mathrm{BT}}$ are given in units of Earth radii. Different symbols represent foreshock cavitons at different cone angles $\left(\theta_{\mathrm{BV}}\right)$. The thick black line is a linear fit to the entire sample. The red dash-dotted line and the blue dashed line are fits for the intermediate ion boundary (Meziane and D'Uston, 1998) and the ULF wave boundary (Greenstadt and Baum, 1986) for cone angles between $40^{\circ}$ and $50^{\circ}$.

for corresponding cone angles are also shown, if they are provided in Greenstadt and Baum (1986) and Meziane and D'Uston (1998). We see from the plots that the fit for a subset of cavitons and the fit to an entire sample match at larger cone angles. In general, when comparing these fits with the calculated intermediate ion and ULF boundaries, it is clear that cavitons always appear further inside the foreshock. The coefficients for all linear fits are provided in Table 2.

\section{Discussion}

In this work we perform a statistical study of 92 foreshock cavitons observed by Cluster 1 during the years 2001-2006. In the spacecraft data the foreshock cavitons appear as regions of diminished values of $B$ and $n$ surrounded by a rim where these two quantities are enhanced compared to the ambient values.

In order for an event to be identified as a caviton, several criteria had to be satisfied: depletions of $B$ and $n$ inside the cavitons had to be deeper than those caused by the surrounding ULF waves; magnetic field and density during the caviton observations must be highly correlated. We show in a case study that the Pearson correlation coefficient for the two quantities during the caviton can be twice as large as during the periods of ULF waves. Cavitons thereby stand out as
Table 2. Coefficients of straight-line fits for foreshock cavitons for different cone angle ranges.

\begin{tabular}{lll}
\hline$\theta_{\mathrm{BV}}$ & $k$ & $n\left(R_{\mathrm{E}}\right)$ \\
\hline All angles & 0.67 & -7.7 \\
$10-20^{\circ}$ & 0.41 & -5.1 \\
$20-30^{\circ}$ & 0.53 & -6.6 \\
$30-40^{\circ}$ & 0.67 & -7.9 \\
$40-50^{\circ}$ & 0.69 & -8.1 \\
$50-60^{\circ}$ & 0.61 & -2.5 \\
\hline
\end{tabular}

distinct structures, different from the ULF background. Also, all cavitons were observed at least five minutes after or before the nearest foreshock compressional boundary (FCB), so that they would not be misidentified with the boundaries. Many foreshock caviton candidates were rejected in the process and this resulted in a relatively small number of events in our sample.

The amplitudes of the depletions in $\boldsymbol{B}$ field and plasma density inside the cavitons are highly correlated (Fig. 9). The average $\Delta B / B$ and $\Delta n / n$ in our sample are 0.47 and 0.52 , respectively. The average duration of the cavitons in the data was $65 \mathrm{~s}$ and their average calculated extents $4.6 R_{\mathrm{E}}$. The majority of the cavitons $(76 / 92,83 \%)$ lasted less than $80 \mathrm{~s}$ and $77 / 92(84 \%)$ had extents less than $6 R_{\mathrm{E}}$.

The longest lasting caviton was observed during $\sim 3 \mathrm{~min}$, and the largest extent was $13 R_{\mathrm{E}}$. The extents were calculated by multiplying the caviton durations by the solar wind speed. A more accurate method would involve calculating the actual caviton velocities by using observations from multiple spacecraft. It was shown by Kajdič et al. (2011) that cavitons propagate sunwards in the SW frame of reference with velocities that are somewhat smaller than the solar wind speed. This would reduce the calculated extents. The measured extents do not represent the actual caviton sizes, since they depend on how a spacecraft actually crosses a caviton. Cavitons may have irregular shapes and the spacecraft may cross them closer to their edges or can penetrate deeper into their interiors.

The hybrid simulations, for example in Blanco-Cano et al. (2011), show that the caviton sizes can vary. In simulations it seems that they become larger as they approach the bow shock. We compared the extents of cavitons in our sample with their distances from the bow shock (not shown). There was no correlation between the two variables.

We show that cavitons appear for a wide range of IMF and SW conditions in the quasi-parallel foreshock, as also suggested by hybrid simulations. However they do not appear for all IMF and SW conditions with the same probability (Fig. 7). 

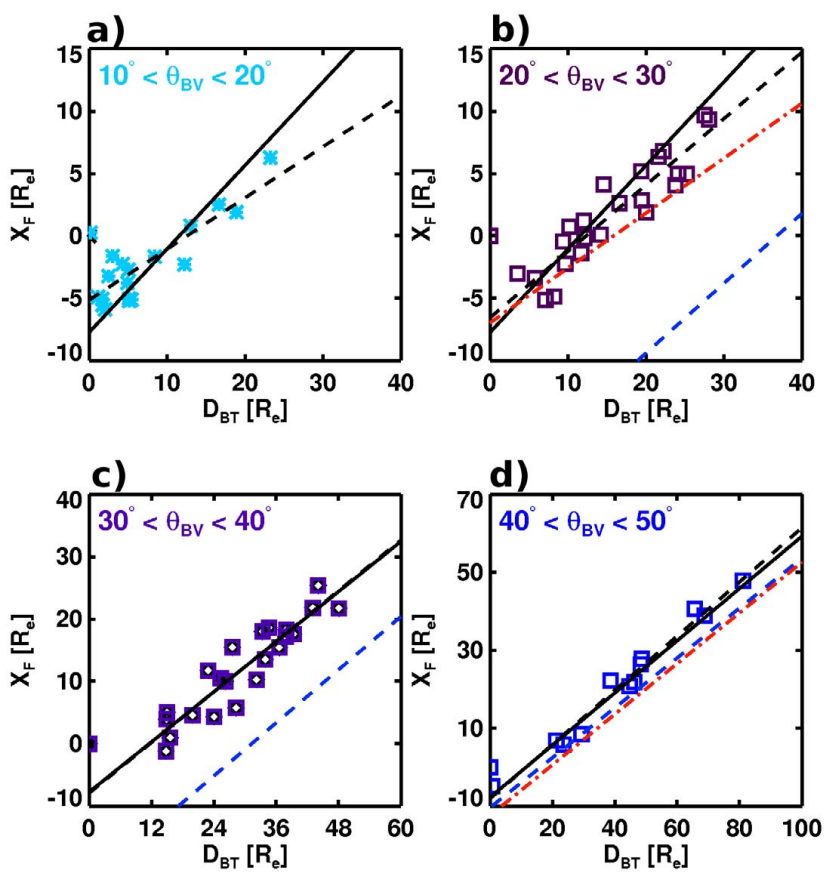

Fig. 12. Positions of foreshock cavitons in solar foreshock coordinates for different cone angles. The black thick line is the same as in Fig. 11 and the black dashed line is a fit to the shown group of cavitons. The red dash-dotted and the blue dashed lines represent intermediate ion and ULF wave boundaries for corresponding cone angles. Note that neither boundary was calculated by Meziane and D'Uston (1998) and Greenstadt and Baum (1986) for $10^{\circ} \leq \theta_{\mathrm{BV}} \leq 20^{\circ}$ and that the ULF wave boundary was also not provided for $30^{\circ} \leq \theta_{B V} \leq 40^{\circ}$.

Cavitons were observed with higher probability for higher $\boldsymbol{B}$ fields, SW velocities, Alfvén speeds and for smaller plasma densities and Alfvénic Mach numbers, when compared to overall SW properties. We have also performed a standard $\chi^{2}$ test with which we calculated the possibility that the differences between the two data sets are due to pure chance. The tests were calculated for distributions of all five quantities. In all cases the $\chi^{2}$ values were very high, giving practically a zero probability that corresponding histograms in the top and middle rows in Fig. 7 show the same distributions and that their apparent differences are purely coincidental.

When we compare caviton locations in the foreshock with intermediate ion and ULF wave boundaries (Figs. 11 and 12), we see that cavitons clearly appear further inside the foreshock. These are the regions populated by compressive ULF fluctuations. In the future it will be interesting to add to these figures the locations of observed foreshock compressional boundaries (FCBs) (Omidi et al., 2009, 2013b; Rojas-Castillo et al., 2013). FCBs separate highly perturbed foreshock plasma from either pristine SW or from the fieldaligned ion beams (FAB) region. Since strong compressive ULF fluctuations are required for the FCB formation, it is likely that the average FCB locations in solar foreshock coordinates will appear just upstream of the average locations of foreshock cavitons.

Several transient phenomena exist in the region upstream of the Earth's quasi-parallel shock. Some of them may exhibit similar signatures in the spacecraft data, so one needs to pay special attention in order to distinguish different structures. In the following paragraphs, we will briefly discuss how cavitons differ from or relate to other phenomena commonly observed in the foreshock region.

It is well known that deep inside the quasi-parallel foreshock, two structures arise from ULF fluctuations: the shocklets and the short-large amplitude magnetic structures (SLAMS) (Hoppe and Russell, 1981; Greenstadt et al., 1995; Scholer et al., 2003; Schwartz and Burgess, 1991; Schwartz, 1991; Schwartz et al., 1992; Giacalone et al., 1993). In the magnetic field data, the shocklets exhibit a compressive character with one steepened, shock-like edge, often accompanied by a whistler wave precursor. Their amplitudes $(\Delta B / B)$ are typically $50 \%$ and their periods range between $\sim 25 \mathrm{~s}$ and $\sim 100 \mathrm{~s}$. SLAMS are the latest stage of evolution of ULF waves. They appear as isolated structures or as embedded inside the long pulsations (LP). SLAMS are regions of enhanced $\boldsymbol{B}$ field, typically between two and five times higher than the average value in the surrounding medium. It has been proposed that shocklets and SLAMS are formed due to the steepening of ULF waves as they pass through the regions of strong suprathermal ion pressure gradients. Eventually they are convected by the SW towards the quasi-parallel bow shock, where they play an important role in its reformation. When compared with the foreshock cavitons, shocklets and SLAMS do not produce depletions in $\boldsymbol{B}$ field and plasma density and SLAMS are observed only very close to the quasi-parallel bow shock. The main difference between the formation mechanisms of SLAMS and shocklets on one side and cavitons on the other is that shocklets and SLAMS arise from steepened ULF waves whereas cavitons form due to interaction of two types of ULF waves - transverse, parallelpropagating and compressive, obliquely propagating fluctuations (Omidi, 2007).

Another phenomenon often observed at the Earth's bow shock are the hot flow anomalies (HFA) (Thomsen et al., 1986; Schwartz et al., 1995; Lucek et al., 2004; Zhang et al., 2010). HFAs occur when a tangential IMF discontinuity interacts with the bow shock. If the conditions are such that the motional electric field on at least one side of the discontinuity points towards it, this field channels the shock reflected suprathermal ions towards the discontinuity and confines them to its immediate vicinity. Such heated plasma then expands and creates depletions of $B$ and $n$ which are surrounded by a rim of enhanced values of the two quantities. There are however several HFA properties that make them easily distinguishable from foreshock cavitons: the plasma inside the HFAs is strongly heated and deviated from its original direction of propagation. Also, inside the HFAs there are 
always IMF discontinuities (current sheets), which is not the case for foreshock cavitons.

Perhaps the phenomena that most resemble the cavitons in the spacecraft data are the foreshock cavities (Sibeck et al., 2001, 2002, 2008; Billingham et al., 2008, 2011; Schwartz et al., 2006). They also exhibit depletions of IMF magnitude and plasma density and are surrounded by enhancements of $B$ and $n$. However these structures are found in part of the foreshock populated by transverse ULF waves and field aligned ion beams or even in the pristine solar wind. The ion populations inside the cavities are hot and thus differ from those in their surrounding regions. The total pressure (solar wind ions + suprathermal ions + electrons + magnetic field) inside them exceeds the surrounding pressure. The two mechanisms that have been proposed for cavities include varying IMF orientations. In the first scenario the surplus total pressure in their interiors causes cavities to expand (total pressure inside the cavitons is the same as in their surrounding thereby, excluding thermal expansion as their formation mechanism, see Kajdič et al., 2011). In another scenario the cavities are just signatures in the spacecraft data due to back and forth motions of the FCBs across the spacecraft. In this case the increased pressure and hot ion populations are observed because the spacecraft briefly enters the highly perturbed section of the foreshock. The global hybrid simulations of the Earth's bow shock also show the foreshock cavitons for any IMF and SW conditions (Omidi, 2007; BlancoCano et al., 2009, 2011), while foreshock cavities appear only in presence of IMF rotations (Omidi et al., 2013b).

\section{Conclusions}

We study the foreshock cavitons observed by the Cluster 1 spacecraft during the years 2001-2006. In order not to misidentify other foreshock phenomena for cavitons, we use stringent criteria in the selection process. Thus only 92 events were included in our sample. The main condition for the formation of the cavitons is the interaction of transverse and compressive ULF waves. These fluctuations are commonly observed upstream of the quasi-parallel section of the Earth's bow shock. We calculate their occurrence rate to be $\sim 2$ events per day. We cannot exclude the possibility that many more cavitons were present in the data but that they were not recognized as such due to their shallow $B$ and $n$ profiles that made them difficult to distinguish from the surrounding ULF background. The statistical results in this paper only apply to those cavitons that evolved sufficiently in order to be included in our sample.

We show that cavitons appear for a wide range of IMF and SW parameters upstream of the quasi-parallel section of the Earth's foreshock. However, cavitons were found preferentially for higher $\boldsymbol{B}$ field, SW velocities, Alfvén speeds and for smaller plasma densities when compared to average SW properties.
Inside the cavitons, the $B$ and $n$ diminished by between $20 \%$ and $\sim 85 \%$ when compared to the ambient values (the lower limit was chosen as one of the selection criteria). The average depletions were $47 \%$ and $52 \%$ for $B$ and $n$, respectively. The magnitudes of $B$ and $n$ depletions were well correlated with the correlation coefficient $K=85 \%$. Their average duration in the data was $65 \mathrm{~s}$ and their average extent was $4.6 R_{\mathrm{E}} .83 \%$ of the cavitons lasted for less than $80 \mathrm{~s}$ and $84 \%$ had extents less than $6 R_{\mathrm{E}}$. The longest lasting cavitons was observed for $\sim 3 \mathrm{~min}$ and its calculated extension was $13 R_{\mathrm{E}}$. The comparison of the cavitons sizes and their distance from the bow shock revealed no correlation between the two quantities.

Additionally we show that foreshock cavitons are not associated with any discontinuities in the IMF and that $B$ and $n$ inside the cavitons are highly correlated, much more than it is the case for the surrounding ULF waves.

We also compare the cavitons with other foreshock phenomena, such as shocklets, SLAMS, HFAs and foreshock cavities and discuss their possible relations. Among the most convincing arguments that show that we correctly identified the foreshock phenomena as foreshock cavitons are the facts that cavitons are not associated with any solar wind plasma heating, flow deflections or IMF discontinuities. The cavitons appear in parts of the foreshock region that are populated by compressive ULF fluctuations. In the future it will be interesting to compare the locations of the cavitons in the solar foreshock coordinates with those of the foreshock compressional boundaries (FCB). We expect that cavitons will appear located just downstream of some average FCB location. This will provide further insight about the complex phenomena in the foreshock region.

Acknowledgements. The authors acknowledge the CL/CLWeb team (http://clweb.cesr.fr), the Automated Multi Dataset Analysis (AMDA) team (http://cdpp-amda.cesr.fr/DDHTML), the Cluster CIS instrument team, the Cluster FGM team (PI Elizabeth Lucek) and the Cluster RAPID team (PI Patrick W. Daly). The work at IRAP was supported by CNRS and CNES. X. Blanco-Cano's work was supported by UNAM-DGAPA-PAPIIT grant IN110511. N. Omidi's work was supported by NSF grant AGS-1007449. Work at UCLA was supported by the NSF under grant ATM04-02213.

Topical Editor C. Owen thanks two anonymous referees for their help in evaluating this paper.

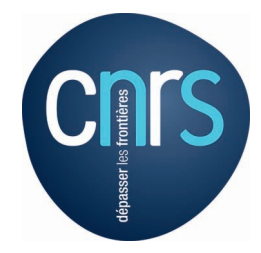

The publication of this article is financed by CNRS-INSU. 


\section{References}

Balogh, A., Carr, C. M., Acuña, M. H., Dunlop, M. W., Beek, T. J., Brown, P., Fornacon, K.-H., Georgescu, E., Glassmeier, K.H., Harris, J., Musmann, G., Oddy, T., and Schwingenschuh, K.: The Cluster Magnetic Field Investigation: overview of in-flight performance and initial results, Ann. Geophys., 19, 1207-1217, doi:10.5194/angeo-19-1207-2001, 2001.

Billingham, L., Schwartz, S. J., and Sibeck, D. G.: The statistics of foreshock cavities: results of a Cluster survey, Ann. Geophys., 26, 3653-3667, doi:10.5194/angeo-26-3653-2008, 2008.

Billingham, L., Schwartz, S. J., and Wilber, M.: Foreshock cavities and internal foreshock boundaries, Planet. Space Sci., 59, 456467, 2011.

Blanco-Cano, X., Omidi, N., and Russell, C. T.: Global hybrid simulations: foreshock waves and cavitons under radial IMF geometry, J. Geophys. Res., 114, A01216, doi:10.1029/2008JA013406, 2009.

Blanco-Cano, X., Kajdič, P., Omidi, N., and Russell, C. T.: Foreshock cavitons for different interplanetary magnetic field geometries: Simulations and observations, J. Geophys. Res., 116, A09101, doi:10.1029/2010JA016413, 2011.

Eastwood, J. P., Lucek, E. A., Mazelle, C., Meziane, K., Narita, Y., Pickett, J., and Treumann, R. A.: The foreshock, Space Sci. Rev., 118, 41-94, doi:10.1007/s11214-005-3824-3, 2005

Giacalone, J., Schwartz, S. J., and Burgess, D.: Observations of suprathermal ions in association with SLAMS, Geophys. Res. Lett., 20, 149-152, 1993.

Greenstadt, E. W. and Baum, L. W.: Earth's compressional foreshock boundary revisited: Observations by the ISEE 1 magnetometer, J. Geophys. Res., 91, 9001-9006, 1986.

Greenstadt, E. W., Le, G., and Strangeway, B.: ULF waves in the foreshock, Adv. Space. Res., 15, 71-84, 1995

Hoppe, M. and Russell, C. T.: On the nature of ULF waves upstream of planetary bow shocks, Adv. Space Res., 1, 327-332, 1981

Hoppe, M. M., Russell, C. T., Frank, L. A., Eastman, T. E., and Greenstadt, E. W.: Upstream Hydromagnetic Waves and Their Association with Backstreaming Ion Populations: ISEE 1 and 2 Observations, J. Geophys. Res., 86, 4471-4492, 1981

Kajdič, P., Blanco-Cano, X., Omidi, N., and Russell, C. T.: Multispacecraft study of foreshock cavitons upstream of the quasiparallel bow shock, Planet. Space Sci., 59, 705-714, 2011

Le, G. and Russell, C. T.: A study of ULF wave foreshock morphology - I. ULF foreshock boundary, Planet. Space. Sci., 40, 1203-1213, 1992a.

Le, G. and Russell, C. T.: A study of ULF wave foreshock morphology - II. Spatial variations of ULF waves, Planet. Space. Sci., 40, 1215-1225, 1992b.

Lin, Y.: Global-scale simulation of foreshock structures at quasi-parallel bow shock, J. Geophys. Res., 108, 1290, doi:10.1029/2003JA009991, 2003.

Lin, Y. and Wang, X. Y.: Three-dimensional global hybrid simulation of dayside dynamics associated with the quasi-parallelbow shock, J. Geophys. Res., 110, A12216, doi:10.1029/2005JA011243, 2005.

Lucek, E. A., Horbury, T. S., Balogh, A., Dandouras, I., and Rème, H.: Cluster observations of hot flow anomalies, J. Geophys. Res., 109, A06206, doi:10.1029/2003JA010016, 2004.
Meziane, K. and d'Uston, C.: A statistical study of the upstream intermediate ion boundary in the Earth's foreshock, Ann. Geophys., 16, 125-133, doi:10.1007/s00585-998-0125-7, 1998.

Narita, Y., Glassmeier, K.-H., Schäfer, S., Motschmann, U., Fränz, M., Dandouras, I., Fornaçon, K.-H., Georgescu, E., Korth, A., Rème, H., and Richter, I.: Alfvén waves in the foreshock propagating upstream in the plasma rest frame: statistics from Cluster observations, Ann. Geophys., 22, 2315-2323, doi:10.5194/angeo-22-2315-2004, 2004.

Omidi, N.: Formation of cavities in the foreshock, AIP Conf. Proc., 932, p. 181, doi:10.1063/1.2778962, 2007.

Omidi, N., Sibeck, D. G., and Blanco-Cano, X.: Foreshock compressional boundary, J. Geophys. Res., 114, A08205, doi:10.1029/2008JA013950, 2009.

Omidi, N., Zhang, H., Sibeck, D., and Turner, D.: Spontaneous hot flow anomalies at quasi-parallel shocks: 2. Hybrid simulations, J. Geophys. Res., 118, 173-180, doi:10.1029/2012JA018099, 2013a.

Omidi, N., Sibeck, D., Blanco-Cano, X., Rojas-Castillo, D., Turner, D., Zhang, H., and Kajdič, P.: Dynamics of the foreshock compressional boundary and its connection to foreshock cavities, J. Geophys. Res., 118, 823-831, doi:10.1002/jgra.50146, 2013 b.

Parks, G. K., Lee, E., Mozer, F., Lin, N., Wilber, M., Lucek, E., Dandouras, I., Rème, H., Mazelle, C., Cao, J. B., Meziane, K., Goldstein, M. L., and Escoubet, P.: Larmor size density holes discovered in the solar wind upstream of Earth's bow shock, Phys. Plasmas, 13, 050701, doi:10.1063/1.2201056, 2006.

Parks, G. K., Lee, E., Mozer, F., Lin, N., Wilber, M., Lucek, E., Dandouras, I., Rème, H., Mazelle, C., Cao, J. B., Meziane, K., Goldstein, M. L., and Escoubet, P.: Density holes in the upstream solar wind, in: 6th Annual International Astrophysics Conference, AIP Conference Proceedings, 932, 9-15, 2008

Rème, H., Aoustin, C., Bosqued, J. M., Dandouras, I., Lavraud, B., Sauvaud, J. A., Barthe, A., Bouyssou, J., Camus, Th., Coeur-Joly, O., Cros, A., Cuvilo, J., Ducay, F., Garbarowitz, Y., Medale, J. L., Penou, E., Perrier, H., Romefort, D., Rouzaud, J., Vallat, C., Alcaydé, D., Jacquey, C., Mazelle, C., d'Uston, C., Möbius, E., Kistler, L. M., Crocker, K., Granoff, M., Mouikis, C., Popecki, M., Vosbury, M., Klecker, B., Hovestadt, D., Kucharek, H., Kuenneth, E., Paschmann, G., Scholer, M., Sckopke, N., Seidenschwang, E., Carlson, C. W., Curtis, D. W., Ingraham, C., Lin, R. P., McFadden, J. P., Parks, G. K., Phan, T., Formisano, V., Amata, E., Bavassano-Cattaneo, M. B., Baldetti, P., Bruno, R., Chionchio, G., Di Lellis, A., Marcucci, M. F., Pallocchia, G., Korth, A., Daly, P. W., Graeve, B., Rosenbauer, H., Vasyliunas, V., McCarthy, M., Wilber, M., Eliasson, L., Lundin, R., Olsen, S., Shelley, E. G., Fuselier, S., Ghielmetti, A. G., Lennartsson, W., Escoubet, C. P., Balsiger, H., Friedel, R., Cao, J.-B., Kovrazhkin, R. A., Papamastorakis, I., Pellat, R., Scudder, J., and Sonnerup, B.: First multispacecraft ion measurements in and near the Earth's magnetosphere with the identical Cluster ion spectrometry (CIS) experiment, Ann. Geophys., 19, 1303-1354, doi:10.5194/angeo19-1303-2001, 2001.

Rojas-Castillo, D., Blanco-Cano, X., Kajdič, P., and Omidi, N.: Foreshock compressional boundaries observed by Cluster, J. Geophys. Res., 118, 698-715, doi:10.1029/2011JA017385, 2013.

Scholer, M., Kucharek, H., and Shinohara, I.: Short large-amplitude magnetic structures and whistler wave precursors in a full- 
particle quasi-parallel shock simulation, J. Geophys. Res., 108, 1273, doi:10.1029/2002JA009820, 2003.

Schwartz, S. J.: Magnetic field structures and related phenomena at quasi-parallel shocks, Adv. Space. Res., 11, 231-240, 1991.

Schwartz, S. J. and Burgess, D.: Quasi-parallel shocks: A patchwork of three-dimensional structures, Adv. Space Res., 11, 231-240, 1991.

Schwartz, S. J., Burgess, D., Wilkinson, W. P., Kessel, R. L., Dunlop, M., and Luhr, H.: Observations of short-large amplitude magnetic structures at a quasi-parallel shock, J. Geophys. Res., 97, 4209-4227, 1992.

Schwartz, S. J.: Hot flow anomalies near the Earth's bow shock, Adv. Space Res., 15, 107-116, 1995.

Schwartz, S. J., Sibeck, D. G., Wilber, M., Meziane, K., and Horbury, T. S.: Kinetic aspects of foreshocks cavities, J. Geophys. Res., 33, L12103, doi:10.1029/2005GL025612, 2006.

Sibeck, D. G., Decker, R. B., Mitchell, D. G., Lazarus, A. J., Lepping, E. P., and Szabo, A.: Solar wind preconditioning in the flank foreshock: IMP 8 observations, J. Geophys. Res., 106, 2167521688, 2001.

Sibeck, D. G., Phan, T. D., Lin, R., Leppinng, R. P., and Szabo, A.: Wind observations of foreshock cavities: a case study, J. Geophys. Res., 107, 1271, doi:10.1029/2001JA007539, 2002.

Sibeck, D. G., Omidi, N., Dandouras, I., and Lucek, E.: On the edge of the foreshock: model-data comparisons, Ann. Geophys., 26, 1539-1544, doi:10.5194/angeo-26-1539-2008, 2008.
Thomsen, M. F., Gosling, J. T., Fuselier, S. A., Bame, S. J., and Russell, C. T.: Hot, diamagnetic cavities upstream from the Earth's bow shock, J. Geophys. Res., 91, 2961-2973, doi:10.1029/JA091iA03p02961, 1986.

Treuman, R. A.: Fundamentals of collisionless shocks for astrophysical application, 1. Non-relativistic shocks, Astron. Astrphys. Rev., 17, 409-535, doi:10.1007/s00159-009-0024-2, 2009.

Wilber, M., Parks, G. K., Meziane, K., Lin, N., Lee, E., Mazelle, C., and Harris, A.: Foreshock density holes in the context of known upstream plasma structures, Ann. Geophys., 26, 37413755, doi:10.5194/angeo-26-3741-2008, 2008.

Zhang, H., Sibeck, D. G., Zong, Q.-G., Gary, S. P., McFadden, J. P., Larson, D., Glassmeier, K.-H., and Angelopoulos, V.: Time history of events and macroscale interactions during substorms observations of a series of hot flow anomaly events, J. Geophys. Res., 115, A12235, doi:10.1029/2009JA015180, 2010.

Zhang, H., Sibeck, D. G., Zong, Q.-G., Omidi, N., Turner, D., and Clausen, L. B. N.: Spontaneous hot flow anomalies at quasiparaallel shocks: 1. Observations, J. Geophys. Res., 118, 3357, doi:10.1002/jgra.50376, 2013 